\title{
ACCIONES PARA LA OBSERVANCIA \\ DE PATENTES ESENCIALES: ¿UNA PRÁCTICA RESTRICTIVA DE LA COMPETENCIA?
}

CARLOS DAVID TAMAYO CÁRdENAS ${ }^{*}$ Y LILIANA GALINDO DÍAZ ${ }^{* *}$

\section{RESUMEN}

El uso de tecnologías estandarizadas es una constante en el mundo contemporáneo que permite disfrutar de la interoperabilidad entre dispositivos.

Tales tecnologías son el resultado de esfuerzos intelectuales susceptibles de protección. Cuando se patentan y adoptan como parte de un estándar, esos esfuerzos dan lugar a una Patente Esencial (SEP) que debe ser utilizada por terceros para cumplir con el estándar y participar en el mercado. Por su naturaleza, las SEP también suponen para su titular el compromiso de licenciar su tecnología bajo términos justos, razonables y no discriminatorios (F/RAND). Sin embargo, ese alcance ha llevado a algunos a argumentar que las demandas por infracción de SEP suponen por sí solas una práctica restrictiva de la competencia. Este artículo expone la manera en que tal enfoque ha sido rechazado por varias cortes alrededor del mundo.

Palabras clave: Patentes esenciales; SEP; infracción; organismos de estandarización; sso, F/RAND.

\footnotetext{
* Abogado de la Universidad Externado de Colombia (2017), especialista en Derecho de la Competencia de la Pontificia Universidad Javeriana (2020), estudiante de la Especialización en Propiedad Industrial, Derechos de Autor y Nuevas Tecnologías de la Universidad Externado de Colombia, (2020). Asociado para el área de Litigios de Patentes de la firma OlarteMoure. Bogotá DC. (Colombia). Correo-e: carlos.tamayo01@est.uexternado.edu.co. Fecha de recepción: 17 de diciembre de 2020. Fecha de aceptación: 27 de enero de 2021. Para citar el artículo: Tamayo Cárdenas, Carlos David y Galindo Díaz, Liliana. "Acciones para la observancia de patentes esenciales: ¿una práctica restrictiva de la competencia?” en Revista de la propiedad inmaterial n.o 31, Universidad Externado de Colombia, enero-julio 2021, pp. 235-270. DoI: https://doi.org/10.18601/16571959.n31.09

** Abogada de la Universidad de los Andes (2011) y especialista en Derecho Comercial de la misma universidad (2013). Coordinadora del área de Litigios de Patentes y Variedades Vegetales de la firma OlarteMoure, Bogotá DC. (Colombia). Contacto: lilianagalindod@gmail.com.
} 


\section{JUDICIAL ENFORCEMENT OF SEP: A LANDSCAPE FOR ANTITRUST CONCERNS?}

\section{ABSTRACT}

The use of standardized technologies is a constant in the contemporary world that allows us to enjoy interoperability between devices.

Such technologies are the result of intellectual endeavours that may be subject of Intellectual Property (IP) rights. If patented and adopted as part of the standard, these endeavours will result into a Standard Essential Patent (SEP) that anyone aiming to participate in the market must use. In addition, given the nature of these patents, the titleholder of a SEP must express a commitment to license its technology under fair, reasonable, and non-discriminatory terms (F/RAND). This scope has led infringers to argue that mere enforcement of SEP involves an antitrust violation. This paper aims to show how several courts around the world have openly rejected this approach.

Keywords: Standard Essential Patents; sEP; Infringement; Standard Setting Organization; sSO; F/RAND.

\section{INTRODUCCIÓN}

La interacción entre propiedad intelectual y derecho de la competencia es un asunto que ha llamado la atención de académicos y estudiosos de una y otra disciplina. Sin lugar a duda, cualquier análisis conceptual sobre la naturaleza de los derechos de propiedad intelectual lleva aparejado un estudio sobre el rol que ocupan en el mercado como "monopolios jurídicos" -y no necesariamente económicos- que permiten excluir a terceros del uso de un signo distintivo, una obra, o una invención, entre otros ${ }^{1}$.

En el campo del Derecho de patentes, durante los últimos años el análisis de esa interacción ha estado enfocado particularmente en el estudio de la existencia y alcance de las denominadas Patentes Esenciales, Standard Essential Patents (sEP, por sus siglas en inglés). Como se explicará en detalle a lo largo de este escrito, las Patentes Esenciales son aquellas que reivindican tecnologías necesarias para cumplir con un determinado estándar de industria ${ }^{2} \mathrm{y}$, por lo tanto, son derechos exclusivos

1 Cfr: Rengifo García, Ernesto. Del abuso del derecho al abuso de la posición dominante, 2. ${ }^{a}$ ed., Bogotá, Universidad Externado de Colombia, 2004, pp. 429-440. La reflexión que plantea el autor sobre la distinción entre el monopolio jurídico y el monopolio económico se explica particularmente en el pie de página 65 de la página 430; Tamayo Cárdenas, Carlos David. Análisis de la responsabilidad civil extracontractual por infracción de patentes de invención: un estudio juridico del daño pecuniario y la función del instituto, Bogotá, Universidad Externado de Colombia, 2017, pp. 130-133.

2 Cfr: Greene, Kyle. L. "Standard Essential Patents and Antitrust Law: Balancing 
de naturaleza especial que protegen invenciones a las que todo agente interesado debe poder acceder para cumplir con el estándar requerido para participar en el mercado que lo ha definido como obligatorio ${ }^{3}$.

Por la especial naturaleza del derecho que ostentan, generalmente los titulares de patentes esenciales están sometidos al cumplimiento de una serie de compromisos contractuales que buscan garantizar que la invención subyacente a su patente está a disposición de quienes necesiten utilizarla para participar en el mercado, a cambio de una contraprestación para el titular ${ }^{4}$. Estas obligaciones, promovidas hoy en día por las mismas organizaciones privadas en las que se lleva a cabo el proceso para la definición de estándares voluntarios de industria ${ }^{5}$, buscan que el titular de la patente -originario o derivado ${ }^{6}$ - esté en disposición de licenciar su derecho a terceros en términos justos, razonables y no discriminatorios (términos F/RAND), o a licenciar dicho derecho en términos razonables y no discriminatorios (términos RAND $)^{8}$, según la política de Propiedad Intelectual adoptada por el organismo de estandarización de que se trate?.

Innovation and Competition", Columbia Business Law Review, vol. 3, diciembre de 2010 , p. 1087. Disponible en [https://journals.library.columbia.edu/index.php/CBLR/article/ view/5120/2370].

3 Son varios los autores que abordan esta necesidad de cumplir con el estándar para participar en el mercado, entre ellos: i) Shapiro, Carl y Lemley, Mark A. "The Role of Antitrust in Preventing Patent Holdup", Stanford Law and Economics Olin Working Paper Series, artículo n. ${ }^{\circ}$ 554, agosto de 2020, p. 2042. Disponible en [https://papers. ssrn.com/sol3/papers.cfm?abstract_id=3666211]; ii) Jones, Alison. "Standard-Essential Patents: Frand Commitments, Injunctions and the Smartphone Wars", European Competition Journal, abril de 2014, vol. 10, p. 21, nota 84. Disponible en [https://www. tandfonline.com/doi/abs/10.5235/17441056.10.1.1]; iii) Suñol Lucea, Aurea. "Patentes y hold-up: la acción de cesación ante compromisos F/RAND", InDret Revista para el Análisis del Derecho, vol. 4, octubre de 2015, p. 11, nota 3. Disponible en [https://dialnet. unirioja.es/servlet/articulo?codigo $=5234391]$.

4 Ídem.

5 Conocidas como Organizaciones de Estandarización, y más comúnmente como Standard Setting Organizaciones (sso por sus siglas en inglés).

6 Este asunto es abordado abiertamente en las Políticas de Propiedad Intelectual de algunas sso, como ocurre por ejemplo en el artículo 6.1bis de la Política de Propiedad Intelectual de ETSI, donde se regula el tema de la transferencia de Patentes Esenciales y la forma en que debe garantizarse que los compromisos F/RAND se mantengan a pesar de la transferencia (European Telecommunications Standards Institute. "Rules of procedure. ETSI Intellectual Property Rights Policy”, ESTI, 2020, p. 40. Disponible en [https://www. etsi.org/images/files/IPR/etsi-ipr-policy.pdf]). En casos de SEP declaradas ante sso que contengan previsiones semejantes, los compromisos F/RAND bien podrían ser considerados como "obligaciones ambulatorias" si es que quisiera clasificárselos bajo las categorías tradicionales de las obligaciones civiles. Para ahondar sobre el tema se sugiere la lectura de Contreras, Jorge L. "Technical standards, standards-setting organizations and intellectual property: a survey of the literature (with an emphasis on empirical approaches)", en Mennel, Peter S. y Schwartz, David. Research Handbooks on the Economics of Intellectual Property Law, vol. 2, Analytical Methods, Northampton Massachusetts, Edward Elgar Publishing, 2019, pp. 38-39. Disponible en [https://papers.ssrn.com/sol3/papers. cfm?abstract_id=2900540].

7 Que corresponde al acrónimo en inglés de las expresiones "Fair, Reasonable and Non-discriminatory".

8 Ídem.

9 Las Políticas de Propiedad Intelectual de las sso delimitan el alcance de los compromisos a los cuales se somete voluntariamente el titular de la Patente Esencial y así, 
Como se intuye, los compromisos $\mathrm{F} / \mathrm{RAND}^{\mathrm{IO}}$ son una herramienta que permite conciliar el derecho exclusivo del titular de la Patente Esencial con la necesidad de los terceros de acceder a la tecnología patentada para cumplir el estándar de industria y así poder participar lícitamente en el mercado sin infringir derechos exclusivos. Sin embargo, y en la medida que tales compromisos no suponen una renuncia a los derechos que de forma natural provienen de la patente en sí, el titular de una Patente Esencial siempre conservará el derecho a perseguir judicialmente a quienes contravengan sus derechos exclusivos ${ }^{11} \mathrm{y}$, particularmente, a demandar por infracción a aquellos que han sido reticentes a contratar una licencia y asumir el pago de las regalías correspondientes, no obstante su intención de seguir beneficiándose ilícitamente de la tecnología patentada para participar en el mercado ${ }^{12}$.

mientras existen algunas que establecen que el deber de licenciamiento debe darse en términos F/RAND, hay otras que limitan ese compromiso de licenciamiento a un parámetro RAND. Como lo han manifestado varias autoridades norteamericanas a través del "Policy Statement on Remedies for Standards-Essential Patents subject to Voluntary F/ Rand Commitments" publicado en 2019, generalmente las sso de ese país acogen el parámetro RAND mientras que las sso de otros países acogen parámetros F/RAND, lo que por supuesto no implica que aquello se trate de un parámetro legal asociado a jurisdicciones específicas pues, se repite, ello depende de la decisión adoptada por la sso de que se trate. En todo caso, y como se sugiere en este mismo documento, es posible hacer uso del término "F/RAND" para referirse indistintamente a los compromisos de licenciamiento asumidos en el marco de una sso cuando el objeto de análisis no verse sobre las diferencias entre una y otra política de licenciamiento, y cuando tales diferencias no incidan directamente sobre el punto a abordar. Así, como quiera que el presente escrito no se enfoca en dichas diferencias, y que ellas tampoco inciden en el análisis de los puntos a abordar, se hará uso del término "F/RAND" para referirse indistintamente al deber de licenciamiento generado en el marco de una sso. Para la revision del document tomado como base de esta consideración cfr. U.S. Patent \& Trademark Office, National Institute of Standards and Technology y U.S. Department of Justice, Antitrust Division. "Policy Statement on Remedies for Standards-Essential Patents subject to Voluntary F/ Rand Commitments", uspto, Nist, Doj. 2019, p. 1. Disponible en [https://www.justice. gov/atr/page/file/1228016/download]. Para analizar los antecedentes jurídicos e históricos de este tipo de compromisos contractuales, se sugiere la lectura de Contreras, Jorge L. -A brief history of F/RAND: analyzing current debates in standard setting and antitrust through a historical lens", Antitrust Law Journal, vol. 80, 2015, n.o 1, pp. 39-120. Disponible en [https://papers.ssrn.com/sol3/papers.cfm?abstract_id=2374983].

10 Ver nota 9.

11 i) Suñol. Op. cit., pp. 15-18; ii) Sidak, Gregory. "Injunctive Relief and the F/rand Commitment in the United States", en Contreras, Jorge L. The Cambridge Handbook of Technical Standardization Law: Competition, Antitrust, and Patents, Cambridge, Cambridge University Press., 2019, pp. 390-392, 404. El artículo del autor también está disponible en [https://www.criterioneconomics.com/docs/injunctive-relief-and-the-frand-commitment. pdf], bajo una paginación distinta; iii) Herrera, Luisa. "Tipologías contractuales en el desarrollo de tecnología: nuevas tendencias hacia la colaboración", Boletín Propiedad Intelectual. Propintel, Universidad Externado de Colombia, 2021. Disponible en [https:// propintel.uexternado.edu.co/nuevas-tipologias-contractuales-en-la-transferencia-de-tecnologia/], entre otros autores.

12 Sobre la forma en que la doctrina coincide en que la acción es procedente contra licenciatarios reticentes o, como se le conoce en derecho anglosajón, contra un "unwilling licensee", cfr. i) Layne-Farrar, Anne. "Why Patent Holdout is not just a fancy name for plain old patent infringement", CPI'S North America Column. Koren W. WongErvin (Global Antitrust Institute), febrero de 2016, p. 4. Disponible en [https://media. crai.com/sites/default/files/publications/Why-Patent-Holdout-is-Not-Just-a-Fancy-Namefor-Plain-Old-Patent-Infringement.pdf]; ii) Langus, Gregor; Lipatov, Vilen y Neven, Damien. "Standard-essential patents: who is really holding up (and when)?, Journal of 
Con frecuencia los infractores de patentes esenciales han intentado defender su posición acudiendo a argumentos aisladamente extraídos del Derecho de la competencia como, por ejemplo, que el titular de una patente esencial no puede sin más solicitar la cesación de una conducta infractora pues, según dicen, incurre en un abuso de la posición de dominio o en cualquier otra práctica restrictiva semejante por el solo hecho de ejercer el más natural de sus derechos al solicitar la cesación de los actos de infracción ${ }^{13}$.

Sin embargo, los últimos pronunciamientos jurisprudenciales relevantes a nivel mundial han demostrado que, así planteada, es una estrategia de defensa llamada al fracaso. Particularmente, tales pronunciamientos han demostrado que el titular de una SEP que ha cumplido con el espíritu de sus compromisos contractuales F/ RAND se encuentra plenamente respaldado por el ordenamiento jurídico y puede por tanto solicitar que el infractor deje de usar la tecnología patentada, entre otras medidas correctivas, al menos hasta que tal infractor normalice su situación contratando una licencia.

El presente escrito busca realizar una explicación de esos pronunciamientos judiciales con el objetivo de demostrar que, al ser ejercidas de manera adecuada, las acciones por infracción de patentes esenciales no solo escapan a los terrenos de las denominadas prácticas restrictivas, sino que además son pro-competitivas en la medida en que, por un lado, protegen los intereses jurídicamente tutelados del titular de la patente, y por otro, evitan que el infractor se beneficie ilícitamente de la tecnología patentada a la manera de un free rider.

Competition Law \& Economics, vol. 9, n.o 2, 2013, p. 3 y ss. Disponible en [https:// papers.ssrn.com/sol3/papers.cfm?abstract_id=2222592]; iii) Recientemente, así lo manifestó también el Departamento de Justicia de Estados Unidos (JOD) a la sso de ese país IEEE: U.S. Department of Justice. Antitrust division, Update to the 2015 Business Review Letter, Request Letter, Institute of Electrical and Electronics Engineers, Incorporated (IEEE). DOJ, septiembre de 2020, pp. 5-6. Disponible en [https://www.justice.gov/atr/ page/file/1315291/download]; iv) Suñol. Op. cit., p. 32.

13 El planteamiento en torno a esta defensa se extrae de los tres principales casos de infracción de SEP analizados en este escrito, a saber: i) Huawei contra ZTE; ii) Sisvel vs. Hair, y iii) Unwired Planet vs. Huawei (citados más adelante), pues en todos ellos una de las principales líneas de defensa del extremo demandado consistió en alegar la supuesta comisión de prácticas restrictivas (abuso de la posición de dominio) asociadas a la interposición de acciones para la observancia de sep. Del lado de la doctrina, esta esta línea de defensa se explica en la obra de autores como: i) Schöler, Karolina. Patents and Standards: The Antitrust Objection as a Defense in Patent Infringement Proceedings, en Waldeck und Pyrmont, Wolrad Prinz zu; Adelman, Martin J., Brauneis, Robert, Drexl, Josef y Nack, Ralph (eds.). Patents and Technological Progress in a Globalized World, Berlin, Springer 2009, pp. 177-194; ii) Suñol. Op. cit., pp. 4-41; iii) VäISÄNEN, Tuire Anninna. "F/RAND Defence in Patent Litigation under German Law”, en VäIsäNEN, Tuire AnniIna. Enforcement of F/RAND Commitments under Article 102 TFEU: The Nature of F/RAND Defence in Patent Litigation, Baden-Baden, Nomos Verlagsgesellschaft mbH, 2011, pp. 65-72. Disponible en [https://www.jstor.org/stable/j.ctv941w6t]; iv) HovenKAMP, Herbert. "F/rand and Antitrust", Cornell Law Review, 2019, vol. 105, pp. 1737-1743. Disponible en [https://scholarship.law.upenn.edu/cgi/viewcontent.cgi?article=3095\&cont ext=faculty_scholarship]. 


\section{LAS DENOMINADAS PATENTES ESENCIALES (SEP)}

\section{I.I. LOS ESTÁNDARES VOLUNTARIOS DE INDUSTRIA}

\section{a. La necesidad de estándares de industria en el marco de un mercado tecnológico globalizado}

Como lo ha manifestado Jorge Contreras, la dinámica internacional de los mercados asociados a las Tecnologías de la Información y las Telecomunicaciones (TIC) exige que los productos fabricados en un país puedan operar fácilmente en otro, de manera que los consumidores finales puedan hacer uso conjunto de sus dispositivos sin necesidad de adecuarlos para satisfacer sus necesidades ${ }^{14}$.

En la práctica, esta necesidad de interoperabilidad tecnológica se traduce en el hecho de que la marca o lugar de fabricación de un producto no suponga un obstáculo para su uso conjunto con dispositivos de un origen diverso, de manera que, por ejemplo, los lectores de este artículo puedan acceder a él en formato PDF sin importar la marca o lugar de fabricación del computador o laptop que utilicen, y sin necesidad de convertir el archivo a un formato diferente para leerlo bajo los sistemas operativos Windows, Linux, o MacOS.

Ese grado de interoperabilidad e interconectividad técnica exigido por un mercado que trasciende las fronteras nacionales se logra a partir de la existencia y adopción generalizada de estándares, definidos como "un conjunto de especificaciones técnicas que proporcionan, o se proponen proporcionar, un diseño común para un producto o procedimiento" ${ }^{15}$. Ahora bien, aun cuando estas especificaciones técnicas pueden tener origen en fuentes diversas (p. ej., la decisión de autoridades regulatorias $)^{16}$, lo cierto es que la doctrina ha identificado que la mayoría de los

14 Son varias las obras en que el autor plantea estas reflexiones: i) Contreras, JorGE L. "National Disparities and Standards-Essential Patents: Considerations for India”, en Bharadwaj, Ashish, Deviah, Vishwas y Gupta, Indranath. Complications and Quandaries in the ICT Sector, Singapur, Springer Open, 2018, p. 1. El libro completo y el artículo incorporado en él se encuentran disponibles en [https://link.springer.com/ book/10.1007\%2F978-981-10-6011-3]; ii) Contreras, Jorge. "Technical standards, standards-setting organizations and intellectual property: a survey of the literature...", cit., p. 4; iii) Contreras, Jorge L. "Introduction", en The Cambridge Handbook of Technical Standardization Law: Competition, Antitrust, and Patents, Cambridge, Cambridge University Press., 2019, pp. 1-2.

15 Suñol. Op. cit., p. 8. La definición que brinda la autora corresponde a la traducción a español de la definición propuesta por el profesor MARK LeMLeY, por lo que se citan ambas fuentes en este punto. En su idioma natal, Lemley define los estándares de la siguiente forma: "I define a standard rather broadly as any set of technical specifications that either provides or is intended to provide a common design for a product or process", contenida en Lemley, Mark A. "Intellectual Property Rights and Standard-Setting Organizations”, California Law Review, vol. 90 (6), University of California, Berkeley, School of Law, diciembre de 2002, p. 1889. Disponible en [https://papers.ssrn.com/sol3/ papers.cfm?abstract_id=310122].

16 Para profundizar en las distintas formas de producción de estándares cfr.: i) BARON, Justus et al. "Making the rules: the governance of standard development organizations and their policies on Intellectual Property Rights", Science for Policy Report, vol. 29655, 
estándares tecnológicos que conocemos y usamos actualmente han tenido origen en procesos de consenso voluntario donde diferentes actores, agrupados bajo Organizaciones de Estandarización (Standard Setting Organizations o sso), definen las especificaciones técnicas que deben marcar el diseño común de un producto o procedimiento ${ }^{17}$.

Los estándares así adoptados se encuentran presentes en la mayoría de recursos que usamos a través de nuestros dispositivos móviles, como ocurre con los sistemas de posicionamiento global GPs, los formatos de compresión de audio y video MP3 y MP4, y los sistemas de comunicación inalámbrica celular imperantes $(4 \mathrm{G})$ y entrantes $(5 \mathrm{G})$, entre muchos otros ${ }^{18}$.

\section{b. Los beneficios derivados de los procesos de estandarización voluntaria}

La definición consensual y voluntaria de estándares a través de las organizaciones de estandarización ha generado múltiples beneficios para la industria y el mercado de las Tic, los cuales han sido ampliamente reconocidos por estudiosos del tema. Así, los académicos han defendido la idea de que los estándares voluntarios: i) promueven la entrada de nuevos actores al mercado, quienes tienen la posibilidad de innovar sobre la base de plataformas tecnológicas ya consolidadas para efectos de "llevar avances tecnológicos a todos los rincones del mundo"19; ii) incrementan las opciones disponibles para los consumidores quienes ven ampliada la oferta de productos existentes en el mercado ${ }^{20}$; iii) fomentan la creación y el desarrollo de economías de red bajo los efectos económicos positivos que de allí se derivan ${ }^{21}$; iv) favorecen la reducción de precios en el mercado ampliando de esa forma la curva de demanda y por tanto el acceso a productos de esta categoría ${ }^{22}$, y v) favorecen la calidad de los productos ofrecidos en el mercado ${ }^{23}$ protegiendo además a los

Joint Research Centre, European Commission, 2019, pp. 24 y ss. Disponible en [https:// publications.jrc.ec.europa.eu/repository/bitstream/JRC1 15004/sdo_governance_final_electronic_version.pdf]; ii) Suñol. Op. cit., p. 8, y iii) Contreras, Jorge. "Technical standards, standards-setting organizations and intellectual property...", cit., p. 4.

17 Ibid., p. 4.

18 Para ver un listado comprensivo de los estándares que existen en la industria de las tic cfr.: i) Contreras, Jorge. "National Disparities and Standards-Essential Patents...", cit., pp. 1, 3; ii) Herrera Sierra, Luisa Fernanda. El derecho de la competencia y las licencias F/RAND: herramientas para el acceso a las invenciones, Bogotá, Universidad Externado de Colombia, 2019, pp. 205-206 (en este punto, la contribución de la autora se limita a la traducción al español del listado de estándares mencionado por Contreras). Para ver una reflexión sobre la relevancia de estos estándares para la industria de las TIC cfr.: iii) Melamed, A. Douglas y Shapiro, Carl. "How antitrust law can make F/rand commitments more effective", The Yale Law Journal, vol. 127, 2017, pp. 2111 y ss. Disponible en [https://digitalcommons.law.yale.edu/cgi/viewcontent.cgi?article $=9300 \&$ context $=y l j]$.

19 Contreras, Jorge. "Introduction", cit., p. 1.

20 i) ídem., p. 11; ii) Shapiro y Lemly. Op. cit., p. 35.

21 Shapiro y Lemly. Op. cit., p. 35.

22 Ibíd., p. 35.

23 Ídem. 
consumidores del riesgo de adquirir productos incompatibles entre $s^{24}$, entre otros efectos positivos.

Así pues, los efectos pro-competitivos de los estándares voluntarios producidos en el marco de organizaciones de estandarización resultan ser una circunstancia notoria que redunda en múltiples beneficios para el consumidor final ${ }^{25}$, lo cual se suma al hecho de que la definición de estándares en estas organizaciones suele obedecer a un proceso de talante participativo y no impositivo en el que los miembros de la organización, esto es, los representantes de la industria, la academia y en ciertas ocasiones del gobierno ${ }^{26}$, eligen por consenso la mejor invención posible para atender una necesidad técnica del sector ${ }^{27}$, siendo esta una invención que, al ser propuesta por uno de los miembros de la organización, generalmente está protegida (o en curso de ser protegida) bajo el sistema tradicional de patentes ${ }^{28}$.

\section{I.2. Patentes esenciales: Definición y Reflexiones}

FRENTE AL CONCEPTO DE ESENCIALIDAD

\section{a. El carácter patentable de las contribuciones técnicas ofrecidas por los miembros de las SSO durante el proceso de estandarización}

$\mathrm{Al}$ agrupar compañías del sector privado e instituciones académicas que en ejercicio de sus actividades generan desarrollos técnicos susceptibles de protección, es natural que los miembros de las organizaciones de estandarización ofrezcan

24 Shapiro, Carl. "Setting compatibility standards: cooperation or collusion?, en Cooper Dreyfuss., Rochelle; Leenheer Zimmerman, Diane y First, Harry. Expanding the boundaries of Intellectual Property, Oxford, Oxford University Press., 2001, p. 88. Disponible en [https://faculty.haas.berkeley.edu/shapiro/standards.pdf].

25 Cfr. particularmente, Shapiro, Carl y Lemley, Mark A. Op. cit., pp. 2049 y ss.

26 Sobre la naturaleza generalmente consensual de los procesos de estandarización, y las particularidades de aquello en contextos relevantes para la industria de las TIC cfr.: i) Gupta, Kirti, Op. cit., pp. 38 y 43 (la autora se centra principalmente en las reglas de 3gpp); ii) Melamed, A. Douglas y Shapiro, Carl. Op. cit., p. 2112 y ss. Sobre la participación de autoridades gubernamentales en los procesos adelantados por las sso cfr.: i) Contreras, Jorge. "Technical standards, standards-setting organizations and intellectual property...", cit., p. 5.

27 Sobre la selección objetiva de soluciones técnicas en el marco del proceso de estandarización y valor intrínseco asociado a tales soluciones inventivas como la mejor tecnología disponible cfr.: i) Padilla, Jorge, Ginsburg, Douglas H. y Wong-Ervin, Koren. "Antitrust Analysis Involving Intellectual Property and Standards: Implications from Economics", Harvard Journal of Law \& Technology, vol. 33, otoño de 2019, p. 17. Disponible en [https://jolt.law.harvard.edu/volumes/volume-33]; ii) Gupta, Kirti. Op. cit., 38; iii) Suñol. Op. cit., p. 13, entre otros.

28 En relación con la existencia de patentes que versan sobre las contribuciones técnicas discutidas en el marco de los procesos de estandarización cfr.: i) Greene. Op. cit., p. 1087 ; ii) Contreras, Jorge. "Technical standards, standards-setting organizations and intellectual property...", cit., p. 9; iii) Contreras, Jorge. "National Disparities and Standards-Essential Patents: Considerations for India”, cit., pp. 5 y ss., y iv) Lemley, Mark A. y Simcoe, Timothy. "How essential are standard-essential patents", Cornell Law Review, vol. 104, 2018, pp. 607 y ss. Disponible en [https://www-cdn.law.stanford.edu/ wp-content/uploads/2019/07/104-Cornell-Law-Review-607-2019.pdf], entre muchos otros. 
sus propios desarrollos para dar solución a las problemáticas que se discuten en el proceso de estandarización, sometiéndolas al escrutinio de sus pares para su incorporación como parte del estándar ${ }^{29}$. Como se intuye, las soluciones propuestas por los miembros de una sso corresponden a respuestas a un problema técnico y, por tanto, coinciden plenamente con el concepto de "invención" alrededor del cual gira el sistema de patentes ${ }^{30}$.

Así, una patente esencial surge cuando una de las invenciones propuestas por el miembro de la organización de estandarización, además de ser exitosamente patentada, resulta adoptada como parte del estándar voluntario de industria que ha construido la organización ${ }^{31}$. La patente esencial, entonces, se hace presente cuando al final del proceso de estandarización resulta que al menos una de sus reivindicaciones concedidas ha quedado incorporada como parte del estándar adoptado por la $\mathrm{sso}^{32}$, de forma que al estar en presencia de esta identidad técnica se está en presencia del nacimiento de una patente esencial ${ }^{33}$.

\section{b. La definición de patente esencial: una aproximación a las nociones de esencialidad técnica y comercial}

Como pudiera anticipar el lector en este punto, actualmente no existe una definición legal de lo que se considera una patente esencial. Por lo tanto, las definiciones que existen son aquellas producidas en el marco de los escenarios donde se lleva a cabo el proceso de estandarización de que se trate, lo cual indica que tampoco existen definiciones unívocas frente al concepto. Lo anterior se ilustra de buena manera en la obra de Timothy S. Simcoe y Allan L. Shampine quienes indican que el concepto de esencialidad (y por tanto el de patente esencial) es usualmente definido por cada sso y por lo mismo, puede variar de una organización de estandarización a otra. Sin embargo, lo anterior no ha sido óbice para que a partir del análisis conjunto de las distintas definiciones estos autores hayan sugerido de

29 Cfr. las mismas referencias contenidas en la nota anterior.

30 Sobre el concepto de invención, cfr. Castro García, Juan David. La propiedad industrial, Bogotá, Universidad Externado de Colombia, 2009, pp. 252-206.

31 Sobre la forma en que surge una patente esencial y el hecho de que ello depende de que lo consignado en el estándar se corresponda efectivamente con lo concedido en el trámite de la patente cfr.: i) Gupta, Kirti. Op. cit., pp. 35-36; ii) Stitzing, Robin, SäÄsilahti, Pekka, Royer, Jimmy y Van Audenrode, Marc. "Over-Declaration of Standard Essential Patents and the Determinants of Essentiality", SSRN Electronic Journal, abril de 2017, p. 4. Disponible en [https://papers.ssrn.com/sol3/papers.cfm?abstract_id=2951617]. y iii) Lemley, Mark A. y Simcoe, Timothy. Op. cit., pp. 616. Estos últimos autores, en particular, exploran cómo la esencialidad final de la patente puede estar mediada por varias circunstancias en el proceso de adopción del estándar y de patentamiento.

32 En ese sentido se pronuncia particularmente el profesor Jorge Contreras al indicar que aun cuando comúnmente se habla de "patentes esenciales", puede ser más preciso referirse a "reivindicaciones esenciales", en tanto una patente puede contener, de hecho, varias reivindicaciones distintas, de manera que al menos una de ellas puede ser esencial al estándar; cfr. Contreras, Jorge L. "Essentiality and Standard-Essential Patents", The Cambridge Handbook of Technical Standardization Law..., cit., pp. 216-219.

33 Cfr. notas 15 y 32 . 
manera sencilla, pero acertada, que una patente esencial es simplemente "aquella requerida para implementar el estándar" 34 .

Como lo indica la doctrina, la principal diferencia entre las definiciones adoptadas por la sso es si la necesidad de la patente para implementar el estándar debe ser valorada desde una perspectiva técnica, o si, por el contrario, debe serlo desde una perspectiva comercial ${ }^{35}$. De forma que si una organización de estandarización decide adoptar la primera de estas alternativas, la patente será considerada una SEP cuando no haya una forma técnica alternativa de cumplir con el estándar que no sea mediante el uso de la patente, mientras que bajo la segunda noción la patente será considerada una SEP cuando corresponda a la forma económicamente rentable (pero no necesariamente la única) de cumplir con el estándar ${ }^{36}$.

La distinción entre esencialidad técnica y comercial no es un asunto menor dado que la definición adoptada por la sso frente a la cual se haya realizado el aporte técnico para la construcción del estándar es, en últimas, lo que debe ser tenido en cuenta como derrotero conceptual para determinar si se está o no en presencia de una patente esencial ${ }^{37}$. En todo caso, de existir una controversia sobre el carácter esencial de una patente, serán los jueces los llamados a decir la última palabra, apoyando sus decisiones en los conceptos técnicos que se produzcan en el marco del proceso judicial correspondiente ${ }^{38}$.

34 Simcoe, Timothy S. y Shampine, Allan L. "Economics of Patents and Standardization: Network Effects, Hold-up, Hold-out, Stacking”, en Contreras, Jorge L. The Cambridge Handbook of Technical Standardization Law..., cit., p. 119.

35 i) Contreras, Jorge L. "Essentiality and Standard-Essential Patents", cit., pp. 217-218; ii) Stitzing, Robin, Sä̈̈skilahti, Pekka, Royer, Jimmy, Van Audenrode, Marc. p. cit., p. 9, y iii) Brachtendorf, Lorenz, Gaessler, Fabian y Harhoff, DiETMar. "Truly Standard-Essential Patents? A Semantics-Based Analysis", CEPR Discussion Papers, 2020, p. 4. Disponible en [https://epub.ub.uni-muenchen.de/74748/1/265.pdf].

36 i) Stitzing, Robin, Sä̈̈smllahti, Pekka, Royer, Jimmy, Van Audenrode, Marc. Op. cit., pp. 9-10; ii) Brachtendorf, Lorenz, Gaessler, Fabian y Harhoff, DietMAr. "Truly Standard-Essential Patents?...", cit., p. 4; iii) Contreras, Jorge L. "Essentiality and Standard-Essential Patents", cit., pp. 217-218. Con acierto los tres autores utilizan la Política de Propiedad Intelectual de etsi como ejemplo de un parámetro de esencialidad técnica, en tanto aquella indica al respecto: "esencial, como término aplicado a los derechos de propiedad intelectual, significa que no es posible en términos técnicos (pero no comerciales), hacer, vender, arrendar, disponer, reparar, usar, o hacer funcionar un equipo o un método que cumpla con el estándar sin infringir ese derecho de propiedad intelectual en cuestión, teniendo en cuenta la práctica técnica regular y el estado del arte generalmente conocido al momento de la estandarización". European Telecommunications Standards Institute (ETSI). "Rules of procedure", cit, pp. 44-45 (trad. libre).

37 Simcoe, Timothy S. y Shampine, Allan. Op. cit., p. 119.

38 i) Ibíd., p. 119; ii) Brachtendorf, Lorenz, Gaessler, Fabian y Harhoff, Dietmar. Op. cit., pp. 3 y ss.; iii) Bekkers, Rudi et al. "Pilot study for essentiality assessment of Standard Essential Patents", en Launch event for Pilot Study for Essentiality Assessment of Standard Essential Patents, European Union, 2020, pp. 29 y ss. Disponible en [https://ec.europa.eu/jrc/en/publication/pilot-study-essentiality-assessment-standardessential-patents]. Particularmente, la última fuente citada ilustra la forma en que las cortes hacen uso de expertos técnicos a la hora de confirmar la esencialidad de la SEP en el marco de un litigio por infracción, en caso de haber posiciones disímiles entre las partes al respecto. 


\section{LOS COMPROMISOS CONTRACTUALES RELACIONADOS}

\section{CON LAS PATENTES ESENCIALES}

Como se ha anticipado, los titulares de patentes esenciales tienen la obligación de cumplir con ciertos compromisos contractuales para evitar prácticas que puedan afectar la adopción de un determinado estándar, o el acceso a la tecnología estandarizada. En ese sentido, las organizaciones de estandarización cuentan con políticas internas que regulan aspectos relacionados con los derechos de propiedad intelectual de sus miembros, y específicamente con sus patentes. Estas políticas regulan la forma como opera el derecho de patentes en el marco del desarrollo de estándares voluntarios de industria, permitiendo que las innovaciones de los miembros de las organizaciones de estandarización sean efectivamente incluidas en los estándares aprobados.

En ese contexto las organizaciones de estandarización pueden establecer políticas basadas en la participación o en el compromiso, las cuales se diferencian principalmente en que bajo las primeras los compromisos de licenciamiento se exigen como requisito de membresía para acceder a la sso, mientras que en las segundas los compromisos surgen a solicitud de la sso durante el proceso de estandarización ${ }^{39}$. Sin embargo, como lo indican algunos estudios, la mayoría de políticas de derechos de propiedad intelectual se basan en compromiso ${ }^{40}$, y establecen dos obligaciones principales: i) la divulgación del derecho de patente sobre la innovación que puede ser adoptada como un estándar, y ii) el compromiso de licenciar dicha patente bajo términos F/RAND según el lineamiento adoptado por la sso de que se trate $e^{41}$.

\section{I. Sobre LA Obligación DE DIVULGaCión DE LA PATENTE ESENCIAL}

El primer compromiso de los miembros de organizaciones de estandarización es divulgar, antes de que se adopte el estándar correspondiente, la existencia de patentes que podrían resultar relevantes o cubrir el estándar a ser adoptado. Las organizaciones de estandarización imponen esta primera obligación con el fin de evitar un problema de oportunismo a través del denominado patent hold-up, es decir, para impedir que el titular de la patente esconda su existencia con el ánimo

39 International Telecommunication Union (ITU). Understanding patents, competition and standardization in an interconnected world, Génova, 2014, pp. 57-58. Disponible en [https://www.itu.int/en/ITU-T/Documents/Manual_Patents_Final_E.pdf].

40 Ibíd., p. 58.

41 Maskus, Keith E. y Merril, Stephen A. Patent Challenges for Standard-Setting in the Global Economy: Lessons from Information and Communications Technology, Washington, National Academy of Sciences, The National Academies Press., 2014, p. 39. Disponible en [https://www.nap.edu/catalog/18510/patent-challenges-for-standard-setting-in-the-globaleconomy-lessons]. Para un entendimiento general sobre este aspecto y la forma en que las organizaciones de estandarización adoptan políticas F/RAND o RAND, se recomienda ver la nota de la página 9. 
de cobrar regalías exorbitantes o demandar intempestivamente por infracción a quienes hayan implementado el estándar tras su aprobación ${ }^{42}$.

El momento para realizar dicha declaración varía dependiendo de la organización de estandarización de que se trate. Sin embargo, por regla general la declaración siempre debe realizarse en un momento anterior a la adopción definitiva del estándar $y$, en todo caso, tan pronto como sea posible, como se desprende de las políticas de propiedad intelectual de varias sso representativas para el mercado de las TIC:

\begin{tabular}{|c|c|}
\hline $\begin{array}{l}\text { El deber de divulgación bajo las políticas de ISO, } \\
\text { IEC y UIT }\end{array}$ & El deber de divulgación bajo las políticas del ETSI \\
\hline $\begin{array}{l}\text { "[...] cualquier parte que participe en el trabajo } \\
\text { de la UIT, ISO o IEC debe, desde el principio, } \\
\text { indicar al director de la UIT-TSB, el director de la } \\
\text { UIT-BR, o las oficinas de los directores ejecutivos } \\
\text { de ISO o IEC, respectivamente, sobre cualquier } \\
\text { patente conocida o solicitud de patente pendiente } \\
\text { conocida, ya sea propia o de otras organizaciones, } \\
\text { aunque la UIT, ISO o IEC no puede verificar la } \\
\text { validez de dicha información"*. }\end{array}$ & $\begin{array}{l}\text { “[...] 4. Divulgación de Derechos de Propiedad } \\
\text { (DPI) [...] 4.1 Sujeto a la Cláusula } 4.2 \text { a conti- } \\
\text { nuación, cada miembro utilizará sus esfuerzos } \\
\text { razonables, en particular durante el desarrollo de } \\
\text { un estándar o especificación técnica donde partici- } \\
\text { pa, para informar a ETsi sobre los DPI esenciales } \\
\text { de manera oportuna. En particular, un miembro } \\
\text { que presente una propuesta técnica para una } \\
\text { especificación estándar o técnica deberá, de buena } \\
\text { fe, llamar la atención de eTsi sobre cualquiera de } \\
\text { los DPI de ese miembro que podrían ser esenciales } \\
\text { si se aprueba la propuesta. } 4.2 \text { Las obligaciones } \\
\text { de conformidad con la Cláusula } 4.1 \text { anterior, } \\
\text { sin embargo, no implican ninguna obligación } \\
\text { para los miembros de realizar búsquedas de DPI. } \\
4.3 \text { Las obligaciones de conformidad con la } \\
\text { Cláusula } 4.1 \text { anterior se consideran cumplidas } \\
\text { con respecto a todos los y futuros miembros de } \\
\text { una familia de patentes si ETsi ha sido informado } \\
\text { de un miembro de esta familia de patentes en un } \\
\text { momento oportuno"**. }\end{array}$ \\
\hline
\end{tabular}

* International Electrotechnical Commission; International Organization for Standardization y International Telecommunication Union. Guidelines for Implementation of the Common Patent Policy for ITU-T/ITU-R/ISO/IEC, Génova, IEC, ISO, ITU, 2018, p. 9 (trad. libre). Disponible en [https://www.itu.int/dms_pub/itu-t/oth/04/04/ T04040000010005PDFE.pdf].

** ETSI, Op. cit., p. 39 (trad. libre).

42 Suñol. Op. cit., p. 10. Sobre la relación entre el patent hold-up y las obligaciones de declaración que buscan evitarlo cfr.: i) Bekkers, Rudi et al. "Disclosure rules and declared essential patents", en National Bureau of Economic Research, 2017, pp. 4 y ss. Disponible en [https://www.nber.org/system/files/working_papers/w23627/w23627.pdf]; ii) Ganglmair, Bernhard, Froeb, Luke M. y Werden, Gregory J. "Patent hold up and antitrust: How a well intentioned rule could retard innovation", The Journal of Industrial Economics, vol. 60, n. ${ }^{\circ}$ 2, 2012, p. 251. Disponible en [https://www.researchgate.net/ profile/Luke_Froeb/publication/237207208_An_Equilibrium_Analysis_of_Antitrust_as_a_ Solution_to_the_Problem_of_Patent_Hold-Up/links/5731126c08ae08415e6a86cf.pdf]. Sobre el fenómeno del hold-up y las aproximaciones que se han dado frente al mismo cfr.: i) Farrell, Joseph et al. "Standard setting, patents, and hold-up", Antitrust Law Journal, vol. 74, n. ${ }^{\circ} 3$, 2007, p. 603-670. Disponible en [https://faculty.haas.berkeley. edu/shapiro/standards2007.pdf], y ii) Galetovic, Alexander y Haber, Stephen. "The Fallacies of Patent-Holdup Theory", Journal of Competition Law \& Economics, vol. 13, n. ${ }^{\circ}$ 1, 2017, p. 1-44. Disponible en [https://academic.oup.com/jcle/article/13/1/1/3060409]. 
De igual forma, de las políticas citadas también se extrae que la comunicación de las patentes o solicitudes de patentes debe ser proporcionada de buena fe, realizando los mejores esfuerzos para ello. Lo anterior ocurre porque, como se extrae de las políticas citadas, los miembros no están obligados a realizar o presentar búsquedas de patentes, y porque las organizaciones de estandarización tampoco están llamadas a verificar la información proporcionada por el titular de la patente, ni a realizar análisis de validez sobre aquellas, de tal suerte que la buena fe es el derrotero que siempre debe guiar la forma en que los miembros de la sso cumplan con esa obligación ${ }^{43}$. En cualquier caso, se aclara que la declaración realizada por el titular de la patente no equivale a una prueba irrefutable de esencialidad, pues en caso de existir controversia al respecto serán los jueces los llamados a decidir sobre el asunto, así como cualquier otro relativo a la patente en litigio, por ejemplo su validez intrínseca ${ }^{44}$.

Para cumplir con el requisito de divulgación de la patente el interesado debe comunicarlo por escrito a través de formularios de declaración, los cuales podrán ser posteriormente consultados públicamente. Algunas organizaciones de estandarización, como el Instituto Europeo de Estándares de Telecomunicaciones (ETSI), requieren que las divulgaciones se realicen sobre patentes o familias de patentes específicas, y no aceptan una declaración de divulgación general en la que se indique un compromiso "frente a cualquier patente esencial" 45 .

Ahora bien, la divulgación de la patente no garantiza que no vaya a existir el denominado patent hold-up, por lo que dicho compromiso usualmente va ligado a una segunda obligación referida a la disposición y declaración del titular de la patente a licenciarla bajo términos F/RAND.

\subsection{Sobre la obligación De COMPROMETERSE A LICENCIAR BAJO TÉRMINOS F/RAND}

Como se mencionó previamente, los compromisos F/RAND permiten conciliar el derecho exclusivo del titular de la patente esencial con la necesidad de los terceros de acceder a la tecnología patentada para cumplir el estándar de industria, sin infringir derechos exclusivos. Por lo anterior, para el caso del titular de la patente, la obligación de divulgación de una patente o solicitud de patente que pueda ser esencial al estándar que se va a adoptar, debe ir acompañada de una declaración o compromiso de licenciar dicha patente bajo términos justos, razonables y no discriminatorios, o bajo términos razonables y no discriminatorios.

43 Sobre la forma en que las políticas de las sso generalmente no obligan a realizar búsquedas de patentes o análisis sobre su validez, cfr. BeKkers, Rudi et al. Op. cit., p. $94,108$.

44 Cfr. nota 38.

45 Maskus, Keith E. y Merril, Stephen A. Op. cit., p. 39. 
Usualmente los compromisos de divulgación y licenciamiento van entrelazados y se incluyen en el mismo formulario de declaración ${ }^{46}$. Es importante aclarar que los titulares de patentes esenciales pueden no estar dispuestos a asumir un compromiso de licenciar su patente bajo los términos fijados por la sso, por lo que una decisión de ese estilo tiene un impacto en la adopción del estándar al hacer necesario que se eliminen las especificaciones técnicas cubiertas por las tecnologías patentadas cuyo titular no pretende licenciar. Sin embargo, de acuerdo con un estudio realizado por la ITU en el 2014 frente este punto, los rechazos a los compromisos impuestos por las sso son verdaderamente marginales ${ }^{47}$.

$\mathrm{Al}$ igual que la divulgación de la patente esencial, para la mayoría de organizaciones de estandarización el compromiso de licenciamiento se debe realizar antes de adoptar el estándar. De igual forma, frente al alcance del compromiso, la mayoría de sso solicitan que el compromiso se realice: i) de forma global, es decir que no haya ningún tipo de limitación territorial frente a la obligación de licenciar, y ii) que este compromiso sea irrevocable durante la vigencia de la patente en cuestión ${ }^{48}$. Asimismo, existen organizaciones como el ETSI que establecen la posibilidad de que el compromiso esté condicionado a la reciprocidad de licenciamiento por quienes implementan la tecnología ${ }^{49}$.

$\mathrm{Al}$ respecto, vale a pena resaltar que el compromiso del titular no es absoluto, en la medida que no se refiere a una supresión del derecho que le otorga la patente a excluir que terceros infrinjan su patente, a pesar de haber recibido una oferta en términos F/RAND y no hayan querido corresponder a dicha oferta. En ese sentido, el compromiso F/RAND supone obligaciones para las dos partes.

\subsection{Los COMPROMisos F/RAND COMO ObLigaCiones DE DOBLE VÍA}

Las políticas de los organismos de estandarización no incluyen una definición clara de lo que se debe entender por F/RAND, dejando cierta libertad de negociación entre las partes ${ }^{50}$. De hecho, y de acuerdo con lo manifestado por autores como la profesora Suñol,

... hay quienes han aplaudido la ausencia de una definición precisa porque así se dota al compromiso FRAND de la flexibilidad necesaria. Dado que estas normas son

46 Cfr., por ejemplo, el formulario de ETSi que aparece en la parte final de la política de PI de esta sso, y que también puede ser descargado en el siguiente link [https://www. etsi.org/intellectual-property-rights].

47 ITU. "Understanding patents, competition and standardization in an interconnected world", cit., p. 88 .

48 Maskus, Keith E. y Merril, Stephen A. Op. cit., p. 39.

49 etsi. Op. cit., p. 45.

50 Para ahondar sobre el entendimiento de este punto pueden consultarse autores como Sidak, J. Gregory. "The meaning of F/rand, part i: Royalties", Oxford Journal of Competition Law and Economics, vol. 9, n. ${ }^{\circ}$ 4, 2013, pp. 931-1055. Disponible en [https://academic.oup.com/jcle/article/9/4/931/918112?login=true]. 
el resultado de una evolución competitiva, no parece descabellado considerar que su indeterminación no es una debilidad, sino una ventaja ${ }^{51}$.

Como se puede evidenciar de la política de organizaciones como el ETSI, las organizaciones de estandarización sólo obligan a los titulares de patentes esenciales a declarar que "están dispuestos" a licenciar sus patentes bajo términos F/RAND ${ }^{52}$, de donde se entiende que dicha declaración no constituye una licencia automática y, por lo tanto, son los actores en este mercado (titular de la patente e interesados en adoptar el estándar) quienes deben negociar la licencia bajo términos F/RAND, sin que esto ocurra en el marco de los organismos de estandarización.

Así, como quiera que asumir compromisos F/RAND no implica una renuncia a los derechos del titular de la patente ni la concesión de un permiso automático de uso $^{53}$, el interesado en implementar el estándar tecnológico también tiene deberes y obligaciones. En caso de que el titular de la patente esencial cumpla con sus deberes F/RAND durante la etapa de negociación, y el posible licenciatario no corresponda a dicha oferta en debida forma, el titular de la patente esencial tendrá la facultad de ejercer sus derechos a través de una acción por infracción, sin que ello implique de ninguna manera un abuso del derecho o una práctica restrictiva de la competencia.

Los deberes del interesado en adoptar el estándar tampoco están definidos en las políticas de las organizaciones de estandarización. Dichos deberes han sido delimitados a través de una línea jurisprudencial asumida por diferentes cortes alrededor del mundo (que se expone con más detalle en el acápite 3.2), bajo la cual se han podido establecer reglas claras que debe cumplir el potencial licenciatario en el marco de una negociación F/RAND, con el fin de evitar el denominado patent hold-out.

El patent hold-out ha sido definido por la doctrina como la práctica tendiente a ignorar las patentes y resistirse a las pretensiones de su titular, dadas las bajas probabilidades de ser declarado como infractor ${ }^{54}$. Específicamente, para el caso

51 Suñol, Op. cit., p. 14.

52 etsi, Op. cit., p. 41. "6. Disponibilidad de Licencias. 6.1. Cuando un DPI ESENCIAL relacionado con un ESTÁNDAR O ESPECIFICACIÓN TÉCNICA en particular se divulga frente a ETSI, el Director General del ETSI solicitará inmediatamente a su propietario para que en un plazo de tres meses indique un compromiso irrevocable por escrito de que está dispuesto a otorgar licencias irrevocables en términos y condiciones justos, razonables y no discriminatorios ('F/RAND') sobre dichos DPI al menos en la siguiente medida” (trad. libre, cursivas fuera de texto).

53 Sobre la forma en que el titular no renuncia a su derecho por el compromiso F/ RAND, cfr. los autores y referencias citados en las notas 10 y 11 . Sobre la forma en que los compromisos F/RAND no suponen licencia implícita o automática cfr.: i) Pentheroudakis, Chryssoula y Baron, Justus. "Licensing terms of standard essential patents: A comprehensive analysis of cases. Science for Policy Report", Joint Research Centre, European Commission, 2017, p. 38. Disponible en [https://publications.jrc.ec.europa.eu/ repository/bitstream/JRC104068/jrc104068\%20online.pdf]; ii) Suñol, Op. cit., p. 16. Sobre la forma en que tampoco corresponden a una licencia obligatoria cfr. Herrera Sierra, Luisa Fernanda. "El derecho de la competencia y las licencias F/RAnd...”, cit., p. 216.

54 Chien, Colleen V. "Holding Up and Holding Out", Michigan Telecommunications 
de SEP, esta práctica se configura cuando los potenciales licenciatarios se resisten o no corresponden a ofertas F/RAND, mientras continúan implementado y haciendo uso libre de la tecnología protegida, basándose en un argumento de comisión de supuestas prácticas restrictivas de la competencia para oponerse a la celebración de un acuerdo.

Sin embargo, como se explica detenidamente a continuación, ya desde el emblemático caso de Huawei vs. ZTE el Tribunal de Justicia de la Unión Europea estableció los lineamientos mínimos que debe cumplir cada una de las partes durante la negociación de una licencia F/RAND manifestando que el posible licenciatario debe: i) expresar su voluntad de obtener una licencia bajo términos F/RAND una vez el titular de la patente esencial le haya notificado sobre la existencia de la misma; ii) responder diligentemente (sin dilaciones injustificadas) a tal oferta bien sea mediante la aceptación de aquella o mediante la presentación de una contraoferta F/RAND y, en todo caso, iii) proporcionar al titular de la patente una seguridad adecuada y suficiente durante la negociación ${ }^{55}$.

\section{AL SER ADECUADAMENTE EJERCIDAS, LAS ACCIONES}

PARA LA PROTECCIÓN DE PATENTES ESENCIALES ESCAPAN

AL ÁMBITO DE LAS PRÁCTICAS RESTRICTIVAS DE LA COMPETENCIA

\section{I. LA TENDENCIA DE LOS INFRACTORES DE PATENTES ESENCIALES \\ A ALEGAR LA COMISIÓN DE PRÁCTICAS RESTRICTIVAS DE LA COMPETENCIA \\ A LA HORA DE DEFENDERSE DE LAS ACCIONES POR INFRACCIÓN}

Como ha tenido oportunidad de manifestarlo la doctrina especializada en la materia, y como se desprende de la lectura de las políticas de propiedad intelectual implementadas por las principales organizaciones de estandarización, el titular de una patente esencial no renuncia a su derecho natural de excluir a terceros del uso no autorizado de su invención patentada, por el hecho de asumir voluntariamente los compromisos F/RAND asociados a aquella ${ }^{56}$. Por lo tanto, es perfectamente posible que el titular de una patente esencial demande a aquellos que hagan uso no autorizado de su invención, y solicite la consecuente cesación de la conducta infractora para impedir que el sujeto no autorizado continúe beneficiándose ilícitamente de ella, en especial allí donde el demandado haya sido reticente a

and Technology Law Review, vol. 21, 2014, p. 5. Disponible en [https://repository.law. umich.edu/mttlr/vol21/iss1/1/].

55 Tribunal de Justicia de la Unión Europea. Sala Quinta. Sentencia del 16 de julio de 2015, proferida en el curso del asunto C-170/13, correspondiente a una cuestión prejudicial planteada por el Landgericht Düsseldorf de Alemania, en el marco del proceso promovido por Huawei Technologies Co. Ltd. contra zTE Corp. y zte Deutschland GmbH. Disponible en [http://curia.europa.eu/juris/document/document.jsf?docid=165911 \&doclang=ES].

56 Cfr. notas 10 y 11 para revisar los múltiples autores que soportan esta afirmación. 
contratar una licencia para poder poner en el mercado productos que cumplan con el estándar de que se trate.

Sin embargo, el análisis de los precedentes que más adelante se relacionan demuestra que a la hora de estructurar su estrategia de defensa la parte infractora suele escudarse en la existencia de estos compromisos, y en la necesidad de hacer uso de la patente esencial para implementar el estándar de industria ${ }^{57}$. Particularmente, el infractor suele estructurar defensas encaminadas a convencer a quien conozca del proceso de que las acciones judiciales promovidas por la contraparte corresponden per se a un abuso de la posición de dominio o una práctica restrictiva y, por lo tanto, el accionante no puede sin más solicitar la cesación de la conducta infractora so pena de materializar cualquiera de esas prácticas.

$\mathrm{Al}$ margen de que dichas alegaciones parten del equívoco de considerar que la titularidad de un derecho de patente equivale de suyo a tener una posición de dominio en el mercado ${ }^{58}$, lo cierto es que durante los últimos años las cortes, tribunales y jueces alrededor del mundo han asumido una línea jurisprudencial que está lejos de avalar la viabilidad de una estrategia de tal tipo.

Concretamente, las autoridades judiciales han defendido la idea de que el titular de una patente esencial no incurre en prácticas restrictivas de la competencia por el simple hecho de reclamar protección sobre su derecho de exclusiva, pues si bien es cierto que en determinadas circunstancias el titular de cualquier derecho de propiedad intelectual puede intentar malograr los objetivos del sistema de protección y afectar el mercado haciéndose responsable de las consecuencias jurídicas que de ello se deriven (incluyendo el fracaso de un proceso judicial), no lo es menos que el titular de una SEP que ha sido responsable en el ejercicio de su derecho y ha cumplido con sus compromisos F/RAND no tiene por qué ser calificado en forma negativa al reclamar protección judicial, frente a un sujeto que ha sido reticente a convenir en una licencia para acceder a la tecnología estandarizada.

Más concretamente, el titular de una SEP que ha estado dispuesto a licenciar su derecho bajo los términos fijados por su sso, debe ser respaldado en forma plena por el mismo ordenamiento jurídico que le ha otorgado el derecho de exclusiva, sin que su solicitud de tutela judicial pueda ser rechazada, o su conducta pueda ser calificada de abusiva, tan solo porque las reivindicaciones de su patente versan sobre una sección del estándar.

Así pues, las decisiones judiciales que se estudian a continuación dan cuenta de esa tendencia judicial a rechazar las estrategias de defensa simplistas planteadas por el infractor desde el campo de las prácticas restrictivas y a proteger al titular de una patente esencial, frente a aquellas infracciones cometidas por usuarios de la tecnología estandarizada que, no obstante su intención de obtener réditos de ella y de beneficiarse de la invención patentada, se han mostrado reacios, reticentes o han empleado tácticas dilatorias a la hora convenir en una licencia que compense

58 Rengifo García, Ernesto. Op. cit., pp. 429-440. 
al titular de la patente por la continua y necesaria explotación de la misma en el mercado.

\subsection{De manera Sistemática las Cortes alRededor Del MUNDO \\ HAN RECHAZADO LA IDEA DE APROXIMARSE A LAS ACCIONES POR INFRACCIÓN DE PATENTES ESENCIALES, ADECUADAMENTE EJERCIDAS, DESDE LA PERSPECTIVA a priori DE LAS PRÁCTICAS RESTRICTIVAS DE LA COMPETENCIA}

Aunque cada uno de los casos que se exponen a continuación presentan complejidades técnicas, jurídicas y económicas que justificarían la realización de un estudio particular y completo para cada uno de ellos, lo cierto es que un análisis de tal naturaleza desbordaría los principales propósitos de este artículo de la manera en que quedaron trazados desde la introducción.

Por lo tanto, tales casos han sido analizados principalmente desde la perspectiva de los argumentos de defensa planteados por la parte demandada bajo la óptica de la supuesta comisión de prácticas restrictivas por la interposición de acciones para la defensa de SEP, sin perjuicio de que puedan ser analizados por el lector desde perspectivas diferentes e incluso más elaboradas que la que aquí se propone.

\section{a. El caso Huawei contra ZTE}

Tanto Huawei como zTE son compañías multinacionales de origen chino dedicadas al desarrollo e implementación de innovaciones dentro de la industria de la tecnología celular móvil. Al tiempo de los hechos, Huawei era titular de una patente declarada ante el ETSI como esencial al estándar de telecomunicaciones móviles LTE, comúnmente conocido como estándar 4G.

Entre noviembre de 2010 y marzo de 2011 Huawei y zTE iniciaron negociaciones encaminadas a licenciar dicha tecnología. En el curso de estas negociaciones, Huawei realizó una oferta de licenciamiento sobre su patente y zTE indicó su intención de negociar una licencia cruzada. A pesar de que dicha licencia nunca se concretó, zTE continuó comercializando productos infractores sin realizar pagos de ningún tipo a favor Huawei.

Por tal motivo, el 18 de abril de 2011 Huawei promovió una acción por infracción de patente ante el Tribunal Regional de Düsseldorf en Alemania, solicitando el cese de la conducta infractora y otras medidas correctivas propias de ese tipo de acción. Como quiera que la defensa de zTE estuvo basada en alegar abuso de posición dominante de Huawei ${ }^{59}$, la corte alemana decidió solicitar al

59 Simpson, Mark y Hidaka, Seiko. "The EU Court of Justice Judgment in Huawei v ZTE: important confirmation of practical steps to be taken by Standard Essential Patent holders before seeking injunctions", Norton Rose Fulbright Publications, 2015. Disponible en [https://www.nortonrosefulbright.com/en/knowledge/publications/8f90efbd/ the-eu-court-of-justice-judgment-in-huawei-v-zte---important-confirmation-of-practicalsteps-to-be-taken-by-standard-essential-patent-holders-before-seeking-injunctions] 
Tribunal de Justicia de la Unión Europea (TJUE) una interpretación prejudicial, planteando varios interrogantes asociados a la aplicación del artículo 102 del Tratado de Funcionamiento de la Unión Europea al caso concreto y, particularmente, interrogantes encaminados a definir las conductas que debe asumir el titular de una patente esencial para evitar que su demanda sea interpretada como restrictiva.

Aunque el caso principal fue desistido por mutuo acuerdo entre las partes ${ }^{60}$, el TJUE alcanzó a emitir una interpretación prejudicial que se ha convertido en un precedente relevante en torno a la valoración de una demanda por infracción de patentes esenciales y, particularmente, en relación con los pasos que, según la decisión, caracterizan una auténtica negociación en términos F/RAND, lo cual supone lo siguiente:

1. En primer término la notificación del uso de la patente al posible licenciatario, en los casos en que este conocimiento no fuese obvio. Es decir, el titular de la patente esencial tiene la obligación de informarle al presunto infractor la existencia y esencialidad de su patente ${ }^{61}$;

2. Posteriormente, el titular de la patente debe presentarle una oferta escrita al posible licenciatario bajo términos F/RAND, la cual debe contener los requisitos mínimos de una oferta e indicar particularmente el precio de la licencia y su fórmula de cálculo ${ }^{62}$;

3. A continuación el presunto infractor tiene la obligación de responder la oferta de una forma diligente y seria, de manera que su respuesta puede consistir o bien en una aceptación de la oferta, o bien en una contraoferta bajos términos F/ RAND. Así, en caso de comprobarse que la respuesta del presunto infractor ha sido dilatoria, irrisoria o infundadamente baja, no se consideraría que una acción por infracción de derechos de patentes sea constitutiva de abuso. Frente a este lineamiento, el Tribunal determinó que no se consideraría como dilatoria la propuesta del presunto infractor de resolver la disputa a través de un arbitraje, aunque en este caso el titular de la patente podría solicitar la constitución de una caución para cubrir los daños que pudieren ocasionarse durante el proceso arbitral ${ }^{63}, y$

4. Posteriormente el potencial licenciatario debe brindarle seguridad al titular de la patente esencial de la seriedad de su intención de negociación, lo cual se puede materializar mediante la constitución de garantías a favor del titular durante

60 Tribunal de Justicia de la Unión Europea. Sala Quinta. Sentencia del 16 de julio de 2015, proferida en el curso del asunto C-170/13, correspondiente a una cuestión prejudicial planteada por el Landgericht Düsseldorf de Alemania, en el marco del proceso promovido por Huawei Technologies Co. Ltd. contra zte Corp. y zte Deutschland GmbH. Sección 39. Disponible en [http://curia.europa.eu/juris/document/document. jsf?docid=165911\&doclang=ES].

61 Ibíd., Sección 71.

62 Ídem.

63 Tribunal de Justicia de la Unión Europea. Sala Quinta. Sentencia del 16 de julio de 2015, cit., Sección 67. 
el tiempo de negociación en un escenario arbitral, o argumentar la invalidez de la patente, por ser esa una respuesta válida frente a una controversia de esa naturaleza ${ }^{64}$.

Del análisis de los lineamientos sentados en esta oportunidad se destaca, principalmente, la forma en que el TJUE definió una serie de pautas de comportamiento que no solo se predican del titular de la patente, sino también del potencial licenciatario, poniendo de presente que aquél no es "víctima" del sistema de patentes, sino que la suya también es una conducta que debe ceñirse a compromisos serios y de buena fe, en caso de tener interés en adoptar un determinado estándar de industria que suponga el uso de una tecnología patentada.

Adicionalmente, dicha decisión puso de presente que el titular de una patente esencial conserva los derechos que aquella le otorga y, por lo tanto, le es posible ejercer acciones por infracción en contra de terceros que no correspondan en debida forma dentro de una negociación F/RAND, lo cual supone que la posición del titular de la SEP no debe ser satanizada y que sus intereses deben ser protegidos judicialmente allí donde se haga evidente que la suya no es una conducta oportunista, o una acción que simplemente haya tomado por sorpresa al usuario de la tecnología.

Sin embargo, a pesar de constituir un precedente de suma relevancia para la materia, la decisión del TJuE planteó serias dudas en lo que a su aplicación práctica se refiere, pues lo intrincado y quizá rígido de los pasos que allí quedaron definidos por el Tribunal dio lugar a cuestionar si aquellos lineamientos deberían ser tenidos como parámetros de aplicación inamovibles, o más bien como simples guías de interpretación adaptables a circunstancias concretas, creando así una suerte de inseguridad jurídica que nunca es bienvenida en el marco de relaciones negociales ${ }^{65}$.

Por fortuna, como se verá en el análisis de los siguientes casos, los parámetros definidos por el TJUE en aquella oportunidad han evolucionado a partir de otras decisiones judiciales tendientes a flexibilizarlos, y poco a poco han brindado claridad respecto de lo que resulta o no reprochable en el marco de la negociación de una licencia F/RAND, y cómo la acción por infracción no debe ser analizada a priori como una práctica restrictiva de la competencia.

\section{b. El caso Sisvel contra Hair}

El 8 de agosto de 2012 la compañía Sisvel adquirió la patente EP 852885 de manos de la empresa Nokia Oy. El 10 de abril de 2013 Sisvel declaró la existencia de esta patente ante el ETSI, y presentó su compromiso irrevocable de estar preparada

64 Ídem.

65 Para un buen resumen de algunas críticas de otros autores a esta decisión, se recomienda la lectura de: Herrera, Luisa. “¿Cuándo la negativa a licenciar debe considerarse anticompetitiva?: comentarios al caso Huawei Technologies vs. zTE Corp.”, Blog de Propiedad Intelectual, Universidad Externado de Colombia, diciembre de 2015. Disponible en [https://propintel.uexternado.edu.co/cuando-la-negativa-a-licenciar-debe-considerarseanticompetitiva-comentarios-al-caso-huawei-technologies-vs.. -zte-corp/]. 
para licenciarla en términos F/RAND, al ser esta una patente esencial al estándar "General Packet Radio Service (GPRS)".

Haier Deutschland GmbH y Haier Europe Trading SRL (referidas en adelante conjuntamente como Haier), son dos compañías dedicadas a la comercialización de celulares y tablets en Alemania que durante septiembre de 2014 ofertaron equipos que infringían la patente de Sisvel en el marco del International Electronics Fair celebrado en Berlín. Como consecuencia de ello Sisvel presentó acción por infracción en contra de Haier, dos años después de haberle notificado por primera vez la ocurrencia de dicha infracción.

$\mathrm{Al}$ asumir su defensa, Haier demandó la validez de la patente objeto de la acción por infracción buscando su anulación judicial, y paralelamente alegó abuso de la posición dominante de su contraparte ante el supuesto ofrecimiento de condiciones que calificó como discriminatorias en comparación con una licencia previamente otorgada sobre la misma tecnología a favor de uno de sus competidores en China.

Mientras que la primera instancia accedió a las pretensiones incoadas por Sisvel, en 2016 la Corte de Apelaciones revocó dicha decisión. Sin embargo, en mayo de 2020 el Tribunal Federal de Justicia de Alemania revocó la decisión de la Corte de Apelaciones aplicando los criterios contenidos en el caso Huawei vs. ZTE.

Como suele ocurrir en los casos de infracción de patentes esenciales, una vez confirmada la esencialidad de la patente respecto del estándar ${ }^{66}$ no hubo discusión alguna sobre la comisión de los actos de infracción ${ }^{67}$. Por ello, el Tribunal centró su análisis en los aspectos jurídicos del caso, y particularmente en determinar si ocurrió un abuso de posición dominante de Sisvel al momento de interponer la acción de infracción en estudio, o si, por el contrario, aquello correspondió a un ejercicio legítimo de sus derechos exclusivos.

Como primera medida, el Tribunal manifestó que la existencia de derechos exclusivos en cabeza del titular de un derecho de propiedad intelectual no es una circunstancia que configure por sí misma una posición de dominio en el mercado ${ }^{68}$, pues realmente dicha posición solamente proviene de un cúmulo de circunstancias y factores que en su mayoría se rigen por conceptos de naturaleza económica ${ }^{69}$. Sin embargo, a renglón seguido reconoció que el titular de una patente esencial

66 Sobre la manera en que las cortes suelen apoyarse en la valoración de dictámenes periciales para determinar la esencialidad de la patente en casos de infracción cfr. Brachtendorf, Lorenz, Gaessler, Fabian y Harhoff, Dietmar. Op. cit., pp. 3 y ss.

67 Sisvel International S.A. vs. Haier Deutschland GmbH. Federal Court of Justice of Germany, вGH. Sentencia del 5 de mayo de 2020. KzR 36/17, p. 19. Disponible en [http://eplaw.org/wp-content/uploads/2020/07/DE-FCJ-Sisvel-v-Haier-English.pdf]. Específicamente sobre el tema de la infracción por el cumplimiento del estándar, el Tribunal estableció para este caso que "una estación móvil que cumpla con el estándar GPRS necesariamente hace uso las funcionalidades de la Reivindicación 12 de la patente en litigio. Como ha declarado el Tribunal de Apelación sin objeción alguna de las partes, tales especificaciones son de cumplimiento obligatorio”.

68 Ibíd., p. 18.

69 Sisvel International S.A. vs. Haier Deutschland GmbH. Federal Court of Justice of Germany, BGH, cit., pp 18 y ss. 
ostenta en efecto una posición estructuralmente superior en el marco de una negociación de una licencia, que se deriva principalmente "de la posibilidad legal de exigir a terceros que no ingresen o mantengan en el mercado productos que resulten conformes a la invención"70.

Ahora bien, y más allá de la configuración de una posición de dominio que bajo las anteriores consideraciones puede presentarse en cabeza del titular de una patente esencial, el Tribunal fue claro en determinar que el solo hecho de ejercer acciones judiciales para la defensa de una patente de tal tipo no constituye un abuso de dicha posición, dado que en ese análisis se debe considerar la actitud de ambas partes frente a la negociación y, en particular, el hecho de que ambas hayan cumplido o no con sus compromisos F/RAND.

Así, por ejemplo, por un lado el Tribunal determinó que habrá un abuso de posición de dominio del titular de la patente cuando haya promovido acciones judiciales a pesar de haber recibido una oferta incondicional bajo verdaderos términos F/RAND, pero por el otro también señaló que el titular de una patente esencial no está vedado de hacer valer judicialmente sus derechos pues solamente debe tolerar el uso de su invención respecto de aquellos que de una u otra forma se encuentran cobijados bajo su consentimiento ${ }^{71}$.

Por lo anterior, al aplicar los lineamientos del caso Huawei vs. ZTE, el Tribunal determinó que Sisvel sí había cumplido con su obligación inicial de notificar a la contraparte sobre la infracción y la posibilidad de suscribir una licencia en términos F/RAND, en la medida que dicha notificación solo debe contener una justificación razonada de la infracción de la patente, sin que ello implique que se deben aportar explicaciones técnicas detalladas sobre la infracción en sí misma. Acerca de los siguientes lineamientos de este precedente, el Tribunal consideró que no se podía establecer que Sisvel hubiese abusado de su posición dominante al no haber realizado una oferta en términos F/RAND antes de promover la acción de infracción, toda vez que dicha oferta debe realizarse basada en la disposición que tuviese el potencial licenciatario de suscribir, en efecto, una licencia.

En este caso, se comprobó que Haier no solo respondió a la oferta un año después de la notificación de infracción, sino que, además, lo hizo para expresar

70 Ibíd., p. 21.

71 Ibíd., pp. 23 y 24. Concretamente, en la decisión se señala lo siguiente: "Por lo tanto, constituye un abuso de la posición dominante el hecho de que el titular de la patente ejerza una acción judicial para la defensa de su derecho solicitando la cesación de la conducta (injunction), la destrucción o el decomiso de productos, a pesar de haber recibido una oferta incondicional por parte del infractor para celebrar una licencia en condiciones a las que el titular de la patente no podría rehusar sin violar la prohibición de abuso o discriminación” (p. 24, trad. libre); “[...] al titular de una patente esencial no se le prohíbe hacer valer su patente en el mercado solicitando una orden cautelar u otro tipo de pretensiones [...]. La esencialidad no cambia el hecho de que el titular de la patente solamente tiene que tolerar el uso de su patente si ha autorizado a la persona que hace uso de sus enseñanzas técnicas, o si de cualquier manera ha dado su consentimiento en cumplimiento del deber de no abusar de su poder de mercado" (p. 23, trad. libre). 
simplemente "la esperanza de entablar una negociación formal entre las partes ${ }^{72 "}$ ". Incluso en comunicaciones posteriores se pudo evidenciar que la parte demandada solicitó información técnica detallada respecto de la posible infracción, denotando así una actitud puramente dilatoria frente a la concreción de una licencia. Los anteriores hechos dieron lugar a concluir que no existía una voluntad seria e incondicional de las sociedades demandadas en suscribir una licencia, por lo que no era posible exigir al titular de la patente esencial que presentase una oferta $\mathrm{F} /$ RAND previa a la acción por infracción, cuando desde el principio su contraparte no estaba realmente dispuesta a celebrar negocio alguno.

Del análisis de la sentencia se puede deducir que la decisión del Tribunal alemán constituye un precedente relevante para la interpretación de los lineamientos sentados en el caso Huawei vs. ZTE, dando a entender que no corresponden a parámetros inamovibles de cumplimiento irrestricto, sino más bien a derroteros conceptuales cuyo propósito y sentido debe ser analizado en cada caso concreto.

Adicionalmente, vale la pena considerar que a través de este fallo el Tribunal alemán contribuyó a la necesidad de superar la errónea asimilación entre el monopolio jurídico y el monopolio económico, siendo enfático también en que incluso en caso de que estas circunstancias confluyan en cabeza del titular de la patente esencial, lo propio no es suficiente para considerar como ilegítimas sus actuaciones procesales o para tacharlas de restrictivas en sí mismas, dado que el solo hecho de ostentar posición de dominio en el mercado no significa que los derechos de un agente económico no deban ser respetados, o que el ordenamiento jurídico no deba ofrecerle garantías en caso de infracción ${ }^{73}$.

Sin embargo, podría decirse que la decisión se quedó corta en lo que a este último aspecto se refiere, bajo el entendido de que en el proceso no se hizo un verdadero análisis económico para declarar la existencia de una posición de dominio, lo que implica que la declaración de aquella frente al caso concreto no obedeció a la práctica de pruebas de naturaleza técnica que naturalmente se deben surtir para que la autoridad judicial pueda soportar conclusiones de tal tipo. Si bien una patente esencial podría brindar una posición de dominio en el mercado, se deben tener en cuenta otros elementos propios de este tipo de mercados para poder determinar si efectivamente se configura dicha posición de dominio como, por ejemplo, si el titular de la patente efectivamente participa en el mercado como fabricante de dispositivos móviles.

En cualquier caso, el razonamiento general de la decisión trae nuevamente de presente que el posible licenciatario en una negociación sobre una patente esencial también tiene obligaciones que debe cumplir si pretende entrar al mercado bajo términos F/RAND, y que de no hacerlo será él quien realmente incurra en conductas contrarias a la libre y leal competencia al hacer uso de tecnología patentada en

72 Sisvel International S.A. vs. Haier Deutschland GmbH. Federal Court of Justice of Germany, Op. cit., p. 32.

73 Ibíd., p. 36. 
detrimento de los derechos del titular de la patente esencial, y en detrimento de otros licenciatarios que sí cumplen con sus compromisos F/RAND y pagan regalías para participar lícitamente en el mercado.

\section{c. El caso Unwired Planet contra Huawei}

El 10 de marzo de 2014 las compañías Unwired Planet International Ltd. y Unwired Planet LLC (en adelante Unwired Planet) presentaron una demanda por infracción de seis de sus patentes concedidas en Reino Unido en contra de las compañías Huawei, Samsung y Google ${ }^{74}$.

Al tiempo de los hechos Unwired Planet contaba con un amplio portafolio de patentes adquiridas de manos de Ericsson, que habían sido declaradas como esenciales a los estándares de telecomunicaciones móviles $2 \mathrm{G}, 3 \mathrm{G}$ y $4 \mathrm{G}$ del ETsI ${ }^{75}$, y estaban por lo tanto sometidas al cumplimiento de compromisos F/RAND ${ }^{76}$. Adicionalmente, las patentes en contienda (cinco de las cuales eran SEP) habían sido previamente licenciadas por Ericsson a Huawei, bajo un contrato que estuvo vigente hasta el $2012^{[77]}$.

En abril de 2014, es decir, con posterioridad al inicio del procedimiento judicial por infracción, Unwired Planet realizó una oferta de licenciamiento a Huawei, Samsung y Google ${ }^{78}$. Las demandadas defendieron sus intereses en el proceso negando los hechos relativos a la infracción y esencialidad de las patentes, cuestionando su validez, y elevando excepciones asociadas a la supuesta comisión de prácticas restrictivas de la competencia ${ }^{79}$. La posición de Huawei frente a este punto consistió en señalar que Unwired Planet no había cumplido a cabalidad con los lineamientos del caso Huawei vs. ZTE al haber hecho una oferta posterior a la demanda y, por tanto, la demandante habría incurrido en un abuso de posición de dominio ${ }^{80}$. Durante los años posteriores al inicio del proceso, tanto Samsung como Google llegaron a un arreglo con Unwired Planet, mientras que Huawei decidió seguir adelante con el litigio.

74 Unwired Planet International Ltd. et al. vs. Huawei Technologies (UK) Co. Ltd. et al. UK Supreme Court. Sentencia del 26 de agosto de 2020, uksc 37. Parágrafo 19, p. 10. Disponible en [https://www.supremecourt.uk/cases/docs/uksc-2018-0214-judgment.pdf].

75 Unwired Planet International Ltd. et al. vs. Huawei Technologies (UK) Co. Ltd. et al. High Court of Justice. Chancery Division. Patents Court. Before Mr. Justice Birss. Sentencia del 5 de abril de 2017, Ewhc 2988 (Pat). Parágrafos 1, 4, p. 3. Disponible en [https://www.twobirds.com/ //media/files/unwired-planet-v-huawei-frand--ewhc-2988--public--final-redactions--fi.pdf?la=en].

76 Ibíd., parágrafos 1, 4, p. 3.; Unwired Planet International Ltd. et al. vs. Huawei Technologies...”, cit., Parágrafo 16, p. 9.

77 Ídem.

78 Unwired Planet International Ltd. et al. vs. Huawei Technologies (UK) Co. Ltd., cit., Parágrafo 5, p. 3.

79 Ibíd., Parágrafos 5 y ss., pp. 3 y ss.

80 Ibíd., Parágrafos 129 y ss., pp. 43 y ss. 
El 5 de abril de 2017, tras resolver previamente los aspectos técnicos del caso relativos a la validez y esencialidad de las patentes ${ }^{81}$, el Juez Birss dictó una sentencia histórica bajo la cual dio la razón a Unwired Planet en varias de sus posiciones, defendiendo la idea de que "no es necesario basarse en el derecho de la competencia para exigir el cumplimiento de compromisos F/RAND". Concretamente, el juez señaló que aun cuando la oferta de licenciamiento realizada por Unwired Planet tuvo lugar de manera posterior al inicio del proceso, lo cierto es que la interposición de la demanda y la solicitud de cesación de la conducta infractora no fue un hecho que tomara por sorpresa a Huawei o que hubiese tenido el objetivo de emboscarla para aceptar una tarifa de licencia, por lo cual la orden de cesación de la conducta propia de la acción por infracción resultaba procedente ${ }^{82}$.

Como era de esperar, la decisión fue cuestionada por Huawei y tras su confirmación por la Corte de Apelaciones y la insistencia del demandado en la revisión de este asunto, el caso llegó a manos de la Corte Suprema de Justicia del Reino Unido que acumuló su estudio con el de otro expediente de circunstancias semejantes. Así, el 26 de agosto de 2020, la Corte Suprema dio a conocer una decisión histórica en la que, además de abordar las principales cuestiones y discusiones que surgen alrededor del litigio de SEP, respaldó en forma vehemente el razonamiento y la aproximación del Juez Birss y de la Corte de Apelaciones en el análisis de las defensas elevadas por los infractores de sep desde el derecho de la competencia.

En particular, la Corte rechazó esa aproximación a priori de la defensa a considerar las acciones por infracción de SEP como una práctica restrictiva, confirmando que tales acciones no implican por sí un abuso de la posición de dominio y que los planteamientos del caso Huawei vs. ZTE constituyen una guía de referencia sobre la forma en que el titular de la patente esencial se puede aproximar al demandado para ofrecer el uso de su tecnología, mas no a un manual de pasos mandatorios e irrestrictos que de no ser seguidos al pie de la letra den lugar a una situación de abuso. Más aún, la Corte aclaró que el propósito de dichas pautas es evitar conductas sorpresivas en las que se pretenda emboscar a "usuarios de buena fe de la tecnología" lo cual no ocurre cuando las circunstancias del caso concreto permiten ver que el demandado se encontraba suficientemente advertido de la infracción.

Finalmente, la Corte tuvo oportunidad de manifestar que en circunstancias como las analizadas las órdenes de cesación de la conducta no solamente son posibles sino también necesarias, pues se muestran como el remedio más eficiente para prohibir la infracción y hacer verdadera justicia, ante la insuficiencia de medidas

81 Particularmente ilustrativos sobre la forma en que la infracción se concreta respecto de cada producto habilitado para el cumplimiento del estándar cobijado por la patente esencial, resultan los razonamientos del Juez Birss a este respecto en Unwired Planet International Ltd. et al. vs. Huawei Technologies (UK) Co. Ltd. et al. High Court of Justice. Chancery Division. Patents Court. Before Mr. Justice Birss. Sentencia del 23 de noviembre de 2015, EwHC 3366 (Pat). Parágrafos 100 y ss., pp. 21 y ss. Disponible en [https://www.bailii.org/ew/cases/EwHC/Patents/2015/3366.html].

82 Unwired Planet International Ltd. et al. vs. Huawei Technologies (UK) Co. Ltd. et al, cit., Parágrafo 807 , conclusiones 17 y 18 , p. 168. 
alternativas que, por ejemplo, solamente se encuentren basadas en la indemnización de daños pecuniarios ${ }^{83}$.

Del análisis de la sentencia se puede deducir que la decisión, tanto de los jueces de instancia como de la Corte Suprema, constituyen un precedente valioso que ayuda a desmitificar la idea de que los pasos de Huawei vs. ZTE corresponden a una camisa de fuerza, y particularmente promueve la idea de que las palabras demanda y abuso no pueden ser tratadas como sinónimos retóricos cuando se trata de la protección de SEP.

Sin embargo, también podría decirse que las decisiones asociadas a este caso pueden ser cuestionadas en cuanto a la falta de análisis económicos para declarar la existencia de una posición de dominio, en la medida en que ese hecho fue dado por sentado sin que realmente se hubiesen practicado pruebas técnicas tendientes a tener por cierta esta dominancia en el mercado ${ }^{84}$.

\section{d. El caso FTC contra Qualcomm}

El 17 de enero de 2017, la Federal Trade Commission (FTC) presentó demanda en contra de la compañía norteamericana Qualcomm Incorporated (Qualcomm), alegando había violado algunas de las disposiciones de la FTC Act y la Sherman Act, con ocasión de la política de licenciamiento de patentes empleada por esta compañía en Estados Unidos.

Al tiempo de los hechos Qualcomm poseía un amplio portafolio de patentes que incluía patentes esenciales para los estándares $3 \mathrm{G}$ (CDMA) y $4 \mathrm{G}$ (LTE), las cuales estaban sometidas al cumplimiento de compromisos contractuales F/RAND definidos bajo las políticas de propiedad intelectual de organizaciones de estandarización como la Asociación de la Industria de Telecomunicaciones (TIA) y la Alianza para Soluciones de la Industria de las Telecomunicaciones (ATIS) ${ }^{85}$. La política comercial de Qualcomm en materia de licenciamiento de SEP consistía en otorgar licencia exclusivamente a favor de los fabricantes de equipos originales y no a favor de otros competidores directos en el mercado de fabricación y comercialización de chips.

Tal política estaba enfocada principalmente en el interés de Qualcomm de obtener el máximo valor posible en la comercialización de sus tecnologías patentadas, evitando una reducción en el valor de aquellas como consecuencia del fenómeno del agotamiento del derecho, pues de licenciar sus SEP a favor de sus competidores en el mercado de chips, sus derechos sobre tales SEP se verían agotados con la venta

83 Ibíd., Parágrafos 166-169, pp. 56-57.

84 De hecho, esta falencia fue reconocida indirectamente por el juez Birss quien señaló en varios de los apartes de su fallo que si en el proceso se hubiesen realizado análisis técnicos adecuados, quizá sus consideraciones frente a la dominancia del demandante habrían sido distintas. Cfr. Unwired Planet International Ltd. et al. vs. Huawei Technologies (UK) Co. Ltd. et al., cit., Parágrafos 670, p. 140, y 807, p. 168.

85 Federal Trade Commission vs. Qualcomm Incorporated. United States Court of Appeals for the Ninth Circuit. Court reference: 19-16122 D.C. n. ${ }^{\circ}$ 5:17-cv-00220-LHK, p. 16. Disponible en [https://cdn.ca9.uscourts.gov/datastore/opinions/2020/08/11/19-16122.pdf]. 
de esos chips por sus competidoras, eliminando así la posibilidad para Qualcomm de licenciar sus patentes a favor de los fabricantes de equipos originales ${ }^{86}$.

En lugar de conceder licencia a sus competidores directos, y teniendo en cuenta que varias de sus SEP debían ser necesariamente utilizadas por aquellos para fabricar y poner en el mercado los chips habilitados para el cumplimiento de los estándares correspondientes, Qualcomm ofrecía a estos fabricantes el compromiso de no entablar contra ellos acciones judiciales por infracción, bajo el compromiso recíproco de estas compañías de no vender chips a favor de fabricantes de equipos originales que a su turno no hubiesen sido previamente licenciados por Qualcomm (es decir, no vender chips a favor de potenciales infractores). Todo lo anterior, bajo una política que, de acuerdo con los expedientes judiciales del caso, era conocida como "no license, no chips" ${ }^{87}$.

El caso fue resuelto en primera instancia por la Corte del Distrito Norte de California, que el 21 de mayo de 2019 acogió en su decisión los principales argumentos expuestos por la $\mathrm{FTC}^{88}$. La decisión, que no generó poca controversia en el sector legal, fue apelada por Qualcomm ante la Corte de Apelaciones del Noveno Circuito de Estados Unidos que el 11 de agosto de 2020 profirió un fallo contundente, reversando la decisión de su inferior y abogando por la necesidad de desligar el cumplimiento de obligaciones F/RAND del campo de las prácticas restrictivas de la competencia.

En efecto, tras estudiar detenidamente las circunstancias fácticas del caso, adentrarse en el análisis de la política de licenciamiento implementada por Qualcomm en Estados Unidos, y revisar de manera objetiva el verdadero efecto de aquella en el mercado de los chips que incorporan las tecnologías estandarizadas $3 \mathrm{G}$ (CDMA) y $4 \mathrm{G}$ (LTE) en ese país, la Corte encontró que ninguna de las alegaciones presentadas por la FTC resultaron verdaderamente probadas en el proceso, y que tanto dicha autoridad como el juez que resolvió el caso en primera instancia habían errado en su aproximación antitrust frente a la controversia.

En efecto, la Corte de Apelaciones encontró que bajo las normas que sancionan las prácticas restrictivas no existe un verdadero deber "en términos de competencia" de que una empresa licencie sus derechos de patente a favor de compañías rivales en el mercado de la fabricación y comercialización de chips y, por tanto, aunque la política de Qualcomm pudiera considerarse "novedosa" en ese aspecto, lo cierto es que la misma no resultaba contraria a la Sherman Act ${ }^{89}$. A partir de allí,

86 Ibíd., pp. 12-13.

87 Ibíd., p. 14.

88 i) Federal Trade Commission vs. Qualcomm Incorporated. United States Court of Appeals for the Ninth Circuit, cit., p. 19; ii) Brumfield, Noah A., EtienneCummings, Shamita y Klaren, Jonathan (Jack). ftc vs. Qualcomm”, en White \& Case Technology Newsflash, mayo de 2019. Disponible en:https://www.whitecase.com/ publications/article/ftc-v-qualcomm\#: : text=On\%20May\%2021\%2C\%202019\%2C\%20 the, $\% 2$ Ddiscriminatory\%20(FRAND)\%20terms].

89 Federal Trade Commission vs. Qualcomm Incorporated. United States Court of Appeals for the Ninth Circuit, cit., pp. 35-36. 
la Corte aclaró que el incumplimiento de compromisos F/RAND, por sí mismo, no es una conducta que genere automáticamente la violación de las normas sobre libre competencia pues frente a ella no opera la aplicación de una regla per se que sugiera lo contrario, en tanto tal circunstancia de incumplimiento contractual debe ser analizada bajo la regla de la razón, siendo carga de la parte demandante demostrar cómo ese simple incumplimiento contractual puede en efecto generar un desbalance tal que afecte la competencia en el mercado ${ }^{90}$.

Para finalizar con tal aproximación, la Corte fue enfática en señalar que el simple incumplimiento de compromisos $\mathrm{F} / \mathrm{RAND}$ no es asunto que deba analizarse bajo el derecho de las prácticas restrictivas de la competencia, en la medida que hay remedios más adecuados para aproximarse a ello, como el derecho de contratos, el equivalente anglosajón a la responsabilidad aquiliana (tort law) y por supuesto, el derecho de patentes ${ }^{91}$.

Respecto de dicha decisión basta con señalar que aun cuando las palabras de la Corte de Apelaciones del Noveno Circuito no fueron emitidas en el marco de una acción por infracción, sino en el marco de una acción por la supuesta comisión de prácticas restrictivas (equivalente entre nosotros a una investigación administrativa), lo cierto es que los planteamientos allí contenidos marcan un derrotero conceptual en cuanto a la valoración que se debe dar a las estrategias de defensa basadas en las alegaciones de ese tipo, y la forma en que los jueces deben aproximarse a tales medios de defensa.

Concretamente, lo manifestado en esta sentencia da a entender que esas defensas no deben corresponder a simples alegaciones carentes de sustento probatorio, pues hablar de prácticas restrictivas en este escenario implica necesariamente demostrar el poder de un agente para definir las condiciones de un mercado y la forma en que el incumplimiento de una simple obligación contractual sería suficientemente para generar una situación patológica dentro del mismo.

Por ello es que, aún de verificarse el incumplimiento de compromisos contractuales impuestos por la sso, este es un asunto que puede ser resuelto desde otras aristas del derecho más adecuadas y, por lo tanto, no debe conducir a pensar automáticamente en la comisión de una práctica restrictiva. Sin lugar a dudas la posición adoptada en esta sentencia se ajusta a esa corriente global por derrumbar varios mitos asociados al litigio de SEP y, entre ellos, el mito de que el infractor es una simple víctima dentro de la controversia.

\subsection{Breves Reflexiones Sobre Los puntos}

EN COMÚN ENTRE LAS DECISIONES ANALIZADAS

El análisis conjunto de las decisiones reseñadas permite ver cómo diferentes cortes alrededor del mundo han desechado las aproximaciones simplistas a considerar que 
la interposición de una acción por infracción de SEP constituye por sí misma un abuso de posición de dominio, o una práctica restrictiva de la competencia. Más aún, esas decisiones han contribuido a la idea de que la titularidad de un derecho de patente no debe ser asimilada automáticamente a una posición de dominio y que, incluso, de demostrarse tal confluencia, el titular conserva un derecho de acción que no puede ser calificado a priori como abusivo.

Los pronunciamientos también muestran que aun cuando el usuario de una tecnología de esa naturaleza tiene derecho a acceder a ella en condiciones justas, razonables y no discriminatorias (o al menos razonables y no discriminatorias), lo cierto es que su actitud frente a las negociaciones para procurarse una licencia de uso también debe ser diligente y ajustada a los parámetros de la buena fe pues, como lo han afirmado algunos, las obligaciones F/RAND son auténticas obligaciones de doble vía ${ }^{92}$.

Así, allí donde el titular de la SEP haya sido diligente en el cumplimiento de sus obligaciones, y su contraparte negocial no haya correspondido a ellas en debida forma, las acciones por infracción no pueden ser consideradas como restrictivas o abusivas. Como se observa en el desarrollo de esta línea jurisprudencial, no existe una forma inequívoca e irrestricta en la que el titular de la SEP deba comportarse para demostrar tal cumplimiento de sus compromisos F/RAND, pues aunque en un primer momento la jurisprudencia parecía haber exigido el seguimiento irrestricto de una fórmula de negociación casi matemática (Huawei vs. ZTE), lo cierto es que esta fórmula ha sido flexibilizada al punto de que, incluso, la presentación de una oferta de licenciamiento previa a la demanda no resulta mandatoria allí donde el usuario de la tecnología haya demostrado desinterés por convenir en el negocio (Sisvel vs. Hair, Unwired Planet vs. Huawei).

En últimas, la línea esbozada por estas autoridades judiciales busca evitar que el usuario infractor eleve ese tipo de defensas para incurrir en un patent hold-out ${ }^{93}$. Por ello, no resulta desacertado decir que las sentencias analizadas encuentran un punto en común en evitar que a través de dichas estrategias procesales los usuarios de mala fe de una tecnología estandarizada se comporten como polizones del mercado tecnológico.

De todo ello llama la atención la forma en que las autoridades judiciales pertenecientes a tradiciones jurídicas distintas (common law y civil law) han llegado a conclusiones semejantes a la hora de aproximarse a un fenómeno que, a pesar del carácter territorial que caracteriza los derechos de patente, tiene profundas implicaciones en el plano internacional.

92 McKool, Jr., Mike et al. "Infringing complaint filed before the United States District Court for The Eastern District of Texas Tyler Division", en el curso de la acción civil 6:12-cv-894, p. 3 Disponible en plataformas como Darts IP.

93 Chien, Colleen V. "Holding Up and Holding Out", Michigan Telecommunications and Technology Law Review, vol. 21, 2014, p. 5. Disponible en [https://repository.law. umich.edu/mttlr/vol21/iss $1 / 1 /]$. 
Más aún, llama la atención que esta aproximación a las defensas basadas en el Derecho de la competencia se presente tanto en sistemas que permiten alegar la invalidez de la patente dentro del proceso por infracción, como en sistemas donde lo propio solo puede hacerse a través de un procedimiento independiente (como ocurre en el caso alemán ${ }^{94}$ ), demostrando así que la facilidad práctica de cuestionar la validez de la patente no es un criterio que deba ser tenido en cuenta para asumir posiciones más o menos laxas en relación con el patent hold-out.

\section{CONCLUSIONES}

La aproximación jurídica y conceptual a los casos por infracción de patentes esenciales constituye todavía un terreno en construcción que, sin lugar a dudas, seguirá ocupando el centro del debate entre aquellos interesados en analizar las múltiples intersecciones que desde la doctrina y la práctica se pueden encontrar en torno a la propiedad intelectual y el Derecho de la competencia.

Inevitablemente, la posibilidad de ejercer derechos exclusivos sobre invenciones que a su turno deben ser utilizadas por múltiples agentes para participar en el mercado, es un asunto que, visto desde una perspectiva aislada, puede parecer controversial. Sin embargo, al adentrarse en el estudio detallado de la materia, ese mismo intérprete puede llegar a percatarse de que necesariamente tal posibilidad está mediada por la existencia de un serie de compromisos contractuales que aseguran que el titular del derecho exclusivo hará buen uso del mismo, al permitir que quienes lo necesiten (y estén dispuestos) puedan acceder a él en términos justos, razonables y no discriminatorios, o términos razonables y no discriminatorios, siendo ese compromiso un justo punto de balance entre ese derecho de exclusión del titular y la necesidad de acceso a la invención por parte de terceros.

Sin embargo, los compromisos F/RAND no implican una renuncia a los derechos de exclusión que de manera natural provienen de la patente, así como tampoco una licencia previa que habilite a cualquiera a aprovecharse de una tecnología que, al quedar estandarizada, fue considerada la mejor forma posible de dar respuesta a un problema técnico para la industria. Por el contrario, como lo ha dicho la doctrina, si hay algo a lo que pudiera considerarse que transige quien voluntariamente se

94 Para una mejor comprensión del sistema bifurcado colombiano y la realización de un comparativo con otras jurisdicciones que, como la británica, manejan un sistema unitario, cfr. i) Galindo DÍaz, Liliana y Tamayo Cárdenas, Carlos David. "La nulidad de la patente como mecanismo de defensa en las acciones por infracción", Blog OlarteMoure, septiembre de 2020. Disponible en [https://www.olartemoure.com/la-nulidad-de-la-patentecomo-mecanismo-de-defensa-en-las-acciones-por-infraccion/; ii) Díaz, Lina M. "La nueva excepción en procesos de infracción”, Boletín Virtual de la Propiedad Intelectual, Propintel, Universidad Externado de Colombia, marzo de 2016. Disponible en [https://propintel. uexternado.edu.co/la-nueva-excepcion-en-procesos-de-infraccion/]. Para el entendimiento del sistema bifurcado alemán se recomienda la lectura de Kellenter, Wolfgang y Migdal, Benedikt. "Patent litigation in Germany: overview", Thomson Reuters, 2020. Disponible en [https://uk.practicallaw.thomsonreuters.com/5-622-3450?transition Type=D efault\&contextData=(sc.Default $) \#$ co_pageContainer]. 
somete a compromisos de esta naturaleza es a cobrar sumas exorbitantes o escandalosas por el uso de su tecnología, sin transigir en algo más que eso ${ }^{95}$.

Por lo tanto, el titular de una patente Esencial que se ha sometido a compromisos F/RAND conserva intacto su derecho a excluir a quien ilícitamente se beneficie de la tecnología estandarizada, y se muestre reticente, dilatorio, o incluso descuidado en obtener una licencia para acceder a ella en términos justos.

Aunque los infractores de patentes esenciales han intentado negar tal posibilidad de acción alegando que abusa de su posición de dominio o incurre en prácticas restrictivas quien, con base en un derecho de tal naturaleza, solicita la cesación en el uso de una tecnología estandarizada, lo cierto es que han sido varios, recientes, y de muy alto nivel los pronunciamientos que han rechazado a priori esa aproximación.

Concretamente, los últimos pronunciamientos en la materia han demostrado que las aproximaciones simplistas a considerar como anticompetitiva la interposición de una acción por infracción de una patente esencial deben ser descartadas de plano, pues aunque no se niega que las situaciones irregulares siempre pueden presentarse en este como en cualquier otro contexto, la verdad es que asumir una posición de tal tipo equivaldría a satanizar el más natural de los derechos derivados de una patente y a afectar el núcleo esencial sobre el que se basa el derecho en sí mismo.

Por ello, diferentes cortes alrededor del mundo han salido en defensa de los titulares de patentes esenciales, para argumentar de manera uniforme y sostenida: i) que el incumplimiento de compromisos F/RAND no es un asunto que por sí mismo deba ser considerado en el campo de las prácticas restrictivas, pues existen otros campos jurídicos más adecuados para abordar los problemas que allí se generen, como son, principalmente, el campo contractual y el de la responsabilidad aquiliana; ii) que en línea con lo anterior, el incumplimiento de compromisos F/ RAND no puede ser considerado per se como una práctica restrictiva, pues cualquier alegación de este tipo debe ser analizada bajo una carga probatoria exigente en la que se demuestren (y no solo se aleguen) verdaderas situaciones patológicas en el mercado; iii) que el ejercicio de acciones judiciales para la defensa de patentes esenciales no implica ni puede ser asimilado a un abuso de la posición de dominio, sobre todo cuando se está en presencia de titulares que han sido diligentes en procurar convenir una licencia y se han encontrado con la dilación o el desinterés de quien simplemente busca beneficiarse de una tecnología estandarizada a la manera de un freerider; iv) que la desidia del usuario de acceder a una licencia para hacer uso legítimo de la invención patentada puede verse en múltiples conductas como, por ejemplo, la existencia de tratativas previas entre las partes que no conducen a negocio alguno, o el hecho de que pese a haber licenciado previamente la tecnología patentada el usuario pretenda seguir haciendo uso de ella sin asumir la carga correspondiente, y, finalmente, v) que en casos de infracción de SEP las 
órdenes de cesación de conducta no solo son posibles sino necesarias para evitar la prolongación de una situación injusta.

Por todo ello, no es desacertado decir que al ser adecuadamente ejercidas bajo los parámetros de la buena fe y el cumplimiento de los compromisos definidos por la sso correspondiente, las acciones para la observancia de SEP escapan al ámbito de las prácticas restrictivas de la competencia, como ocurre con cualquier reclamación judicial justa y adecuadamente formulada para defender un interés lícito jurídicamente tutelado.

\section{REFERENCIAS Y FUENTES CONSULTADAS}

\section{REFERENCIAS}

BARÓN, Justus et al. "Making the rules: the governance of standard development organizations and their policies on Intellectual Property Rights", Science for Policy Report, vol. 29655, Joint Research Centre, European Commission, 2019.

Bekkers, Rudi et al. Disclosure rules and declared essential patents", National Bureau of Economic Research, 2017.

Bekkers, Rudi et al. "Pilot study for essentiality assessment of Standard Essential Patents", en Launch event for Pilot Study for Essentiality Assessment of Standard Essential Patents, European Union, 2020.

Brachtendorf, Lorenz, Gaessler, Fabian y Harhoff, Dietmar. "Truly Standard-Essential Patents? A Semantics-Based Analysis", CEPR Discussion Papers, 2020.

Brumfield, Noah A., Etienne-Cummings, Shamita y Klaren, Jonathan (Jack). FTC vs. Qualcomm", White \& Case Technology Newsflash, mayo de 2019.

Castro García, Juan David. La propiedad industrial, Bogotá, Universidad Externado de Colombia, 2009.

Chien, Colleen V. "Holding Up and Holding Out", Michigan Telecommunications and Technology Law Review, 2014, vol. 21.

Contreras, Jorge L. "A brief history of F/RAND: analyzing current debates in standard setting and antitrust through a historical lens", Antitrust Law Journal, vol. 80, n. ${ }^{\circ} 1,2015$.

Contreras, Jorge L. "Introduction", en The Cambridge Handbook of Technical Standardization Law: Competition, Antitrust, and Patents, Cambridge, Cambridge University Press, 2019.

Contreras, Jorge L. "National Disparities and Standards-Essential Patents: Considerations for India", en Bharadwaj, Ashish, Deviah, Vishwas y Gupta InDRANATH. Complications and Quandaries in the ICT Sector, Singapur, Springer Open, 2018.

Contreras, Jorge L. "Technical standards, standards-setting organizations and intellectual property: a survey of the literature (with an emphasis on empirical 
approaches)", en Mennel, Peter S. y Schwartz, David. Research Handbooks on the Economics of Intellectual Property Law, vol. 2, Analytical Methods, Northampton Massachusetts, Edward Elgar Publishing, 2019.

Díaz, Lina M. "La nueva excepción en procesos de infracción", Boletín Virtual de la Propiedad Intelectual, Propintel, Bogotá, Universidad Externado de Colombia, marzo de 2016.

European Telecommunications Standards Institute (etsi). "Rules of procedure”, ETSI Intellectual Property Rights Policy, ETSI, 2020.

Farrell, Joseph et al. "Standard setting, patents, and hold-up", en Antitrust Law Journal, vol. 74, 2007, n. ${ }^{\circ} 3$.

Galetovic, Alexander y Haber, Stephen. "The Fallacies of Patent-Holdup Theory”, Journal of Competition Law \& Economics, vol. 13, n. ${ }^{\circ}$ 1, 2017.

Galindo DÍaz, Liliana y Tamayo Cárdenas, Carlos David. "La nulidad de la patente como mecanismo de defensa en las acciones por infracción”, Blog OlarteMoure, septiembre de 2020.

Ganglmair, Bernhard, Froeb, Luke M. y Werden, Gregory J. "Patent hold up and antitrust: How a well-intentioned rule could retard innovation", The Journal of Industrial Economics, vol. 60, n. ${ }^{\circ}$ 2, 2012.

Greene, Kyle L. "Standard Essential Patents and Antitrust Law: Balancing Innovation and Competition", Columbia Business Law Review, vol. 3, diciembre de 2010 .

Herrera Sierra, Luisa Fernanda. El derecho de la competencia y las licencias F/ RAND: herramientas para el acceso a las invenciones, Bogotá, Universidad Externado de Colombia, 2019.

Herrera Sierra, Luisa Fernanda. “¿Cuándo la negativa a licenciar debe considerarse anticompetitiva?: comentarios al caso Huawei Technologies vs. ZTE Corp.”, Blog de Propiedad Intelectual, Universidad Externado de Colombia, diciembre de 2015.

Herrera Sierra, Luisa Fernanda. "Tipologías contractuales en el desarrollo de tecnología: nuevas tendencias hacia la colaboración”, Boletín Propiedad Intelectual, Propintel. Universidad Externado de Colombia, febrero de 2021.

Hovenkamp, Herbert. "F/RAnd and Antitrust", Cornell Law Review, vol. 105, 2019.

International Telecommunication Union (ITU). Understanding patents, competition and standardization in an interconnected world, Génova, ITU, 2014.

International Electrotechnical Commission; International OrganizaTION FOR STANDARDiZation e InTERnational Telecommunication Union. Guidelines for Implementation of the Common Patent Policy for ITU-T/ITU-R/ISO/ IEC, Génova, IEC, ISO, ITU, 2018

Jones, Alison. "Standard-Essential Patents: F/Rand Commitments, Injunctions and the Smartphone Wars", European Competition Journal, vol. 10, abril de 2014. 
Kellenter, Wolfgang y Migdal, Benedikt. Patent litigation in Germany: overview, Thomson Reuters, 2020.

Langus, Gregor, Lipatov, Vilen y Neven, Damien. "Standard-essential patents: who is really holding up (and when)?, Oxford Journal of Competition Law \& Economics, vol. 9, n. ${ }^{\circ} 2,2013$.

Layne-Farrar, Anne. "Why Patent Holdout is not just a fancy name for plain old patent infringement", en CPI North America Column, Koren W. Wong-Ervin (Global Antitrust Institute), febrero de 2016.

Lemley, Mark A. "Intellectual Property Rights and Standard-Setting Organizations", California Law Review, University of California, Berkeley, School of Law, vol. 90 (6), diciembre de 2002.

Lemley, Mark A. y Simcoe, Timothy. "How essential are standard-essential patents", Cornell Law Review, vol. 104, 2018.

Maskus, Keith E. y Merril, Stephen A. Patent Challenges for Standard-Setting in the Global Economy: Lessons from Information and Communications Technology, Washington, National Academy of Sciences, The National Academies Press, 2014.

Melamed, A. Douglas y Shapiro, Carl. "How antitrust law can make F/rand commitments more effective", The Yale Law Journal, 2017.

McKool Jr., Mike et al. Infringing complaint filed before the United States District Court for The Eastern District of Texas Tyler Division, en el curso de la acción civil 6:12-cv-894.

Padilla, Jorge, Ginsburg, Douglas H. y Wong-Ervin, Koren. "Antitrust Analysis Involving Intellectual Property and Standards: Implications from Economics", Harvard Journal of Law \& Technology, vol. 33, otoño de 2019.

Pentheroudakis, Chryssoula y Baron, Justus. "Licensing terms of standard essential patents: A comprehensive analysis of cases", Science for Policy Report, Joint Research Centre, European Commission, 2017.

Rengifo García, Ernesto. Del abuso del derecho al abuso de la posición dominante, 2. a ed., Bogotá, Universidad Externado de Colombia, 2004.

Schöler, Karolina. "Patents and Standards: The Antitrust Objection as a Defense in Patent Infringement Proceedings", en Waldeck und Pyrmont, Wolrad Prinz zu, Adelman, Martin J., Brauneis, Robert, Drexl, Josef y Nack, Ralph (eds.). Patents and Technological Progress in a Globalized World, Berlin, Springer, 2009.

Shapiro, Carl y Lemley, Mark A. "The Role of Antitrust in Preventing Patent Holdup", Stanford Law and Economics Olin Working Paper Series, agosto de 2020.

Shapiro, Carl. "Setting compatibility standards: cooperation or collusion?", en Cooper Dreyfuss, Rochelle y Leenheer Zimmerman, Diane y First, Harry. Expanding the boundaries of Intellectual Property, Oxford, Oxford University Press, 2001. 
Sidak, J. Gregory. "Injunctive Relief and the F/rand Commitment in the United States", en Contreras, Jorge L. The Cambridge Handbook of Technical Standardization Law: Competition, Antitrust, and Patents, Cambridge, Cambridge University Press, 2019.

SidAk, J. Gregory. "The meaning of F/RAnd", part i, "Royalties", Oxford Journal of Competition Law and Economics, vol. 9, n. ${ }^{\circ}$ 4, 2013.

Simcoe, Timothy S. y Shampine, Allan L. "Economics of Patents and Standardization: Network Effects, Hold-up, Hold-out, Stacking”, en Contreras, Jorge L. The Cambridge Handbook of Technical Standardization Law: Competition, Antitrust, and Patents, Cambridge, Cambridge University Press, 2019.

Simpson, Mark y Hidaka, Seiko. "The EU Court of Justice Judgment in Huawei vs. ZTE. Important confirmation of practical steps to be taken by Standard Essential Patent holders before seeking injunctions", Norton Rose Fulbright Publications, 2015.

Stitzing, Robin, Sä̈̈skilahti, Pekka, Royer, Jimmy y Van Audenrode, Marc. "Over-Declaration of Standard Essential Patents and the Determinants of Essentiality", en SSRN Electronic Journal, abril de 2017.

Suñol Lucea, Aurea. "Patentes y hold-up: la acción de cesación ante compromisos F/RAND”, InDret Revista para el Análisis del Derecho, vol. 4, octubre de 2015.

Tamayo Cárdenas, Carlos David. Análisis de la responsabilidad civil extracontractual por infracción de patentes de invención: un estudio jurídico del daño pecuniario y la función del instituto, Bogotá, Universidad Externado de Colombia, 2017.

U.S. Department of Justice. Antitrust Division. Update to the 2015 Business Review Letter, Request Letter: Institute of Electrical and Electronics Engineers, Incorporated (IEEE), DOJ, septiembre de 2020.

U.S. Patent \& Trademark Office, National Institute of Standards and Technology y U.S. Department of Justice, Antitrust Division. Policy Statement on Remedies for Standards-Essential Patents subject to Voluntary F/Rand Commitments, USPTO, NIST, DOJ, 2019.

Väisänen, Tuire Annina. "F/RAnd Defence in Patent Litigation under German Law”, en Väisänen, Tuire AnNina. Enforcement of F/RAND Commitments under Article 102 TFEU: The Nature of F/RAND Defence in Patent Litigation, BadenBaden, Nomos Verlagsgesellschaft mbH, 2011.

\section{DeCisiones JUdiciales}

Federal Trade Commission vs. Qualcomm Incorporated. United States Court of Appeals for the Ninth Circuit. Court reference: 19-16122 D.C. n. ${ }^{\circ}$ 5:17-cr00220-LHK.

Tribunal de Justicia de la Unión Europea (TJUE). Sala Quinta. Sentencia del 16 de julio de 2015. Proferida en el curso del asunto C-170/13, correspondiente a una cuestión prejudicial planteada por el Landgericht Düsseldorf de Alemania, 
en el marco del proceso promovido por Huawei Technologies Co. Ltd. contra zTE Corp. y zTe Deutschland GmbH.

Unwired Planet International Ltd. et al. vs. Huawei Technologies (UK) Co. Ltd. et al. UK Supreme Court. Sentencia del 26 de agosto de 2020, UKSC 37.

Unwired Planet International Ltd. et al. vs. Huawei Technologies (UK) Co. Ltd. et al. High Court of Justice. Chancery Division. Patents Court. Before Mr. Justice Birss. Sentencia del 5 de abril de 2017, EwHC 2988 (Pat).

Unwired Planet International Ltd. et al. vs. Huawei Technologies (UK) Co. Ltd. et al. High Court of Justice. Chancery Division. Patents Court. Before Mr. Justice Birss. Sentencia del 23 de noviembre de 2015, ewhC 3366 (Pat).

Sisvel International S.A. vs. Haier Deutschland GmbH. Federal Court of Justice of Germany (BGH). Sentencia del 5 de mayo de 2020, кZR 36/17. 